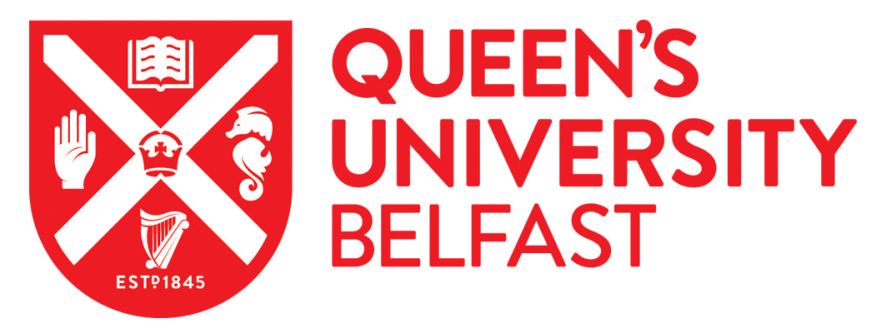

\title{
Fair cake-cutting in practice
}

Kyropoulou, M., Ortega, J., \& Segal-Halevi, E. (2019). Fair cake-cutting in practice. In Fair Cake-Cutting in Practice Proceedings of the 2019 ACM Conference on Economics and Computation.

https://doi.org/10.1145/3328526.3329592

Published in:

Fair Cake-Cutting in Practice

Document Version:

Peer reviewed version

Queen's University Belfast - Research Portal:

Link to publication record in Queen's University Belfast Research Portal

Publisher rights

Copyright 2019 ACM. This work is made available online in accordance with the publisher's policies. Please refer to any applicable terms of use of the publisher.

\section{General rights}

Copyright for the publications made accessible via the Queen's University Belfast Research Portal is retained by the author(s) and / or other copyright owners and it is a condition of accessing these publications that users recognise and abide by the legal requirements associated with these rights.

Take down policy

The Research Portal is Queen's institutional repository that provides access to Queen's research output. Every effort has been made to ensure that content in the Research Portal does not infringe any person's rights, or applicable UK laws. If you discover content in the Research Portal that you believe breaches copyright or violates any law, please contact openaccess@qub.ac.uk. 


\title{
Fair Cake-Cutting in Practice
}

\author{
SUBMISSION 148
}

Using a lab experiment, we investigate the real-life performance of envy-free and proportional cake-cutting procedures with respect to fairness and preference manipulation. We find that envy-free procedures, in particular Selfridge-Conway, are fairer and also are perceived as fairer than their proportional counterparts, despite the fact that agents very often manipulate them. Our results support the practical use of the celebrated Selfridge-Conway procedure, and more generally, of envy-free cake-cutting mechanisms.

We also find that subjects learn their opponents' preferences after repeated interaction and use this knowledge to improve their allocated share of the cake. Learning reduces truth-telling behavior, but also reduces envy.

\section{INTRODUCTION}

The problem of how to fairly divide a divisible good among agents who value different parts of it distinctly has been thoroughly studied in many areas of science over the last seventy years. The heterogeneous good is often referred to as the cake [55], and thus this problem is known as cake-cutting (see 11, 53 and 46 for textbook references). This problem has multiple real-life applications, which include the division of land, inheritances, and cloud computing. Although fundamental breakthroughs have been achieved on the construction of fair cake-cutting procedures, the question of which procedure is fairer in practice has not yet been tackled. This paper reports the results of an experiment that provides useful insights to help answer this question.

Let us start by clarifying what we mean by fair. Although several notions of fairness have been proposed, two important ones stand out for their intuitive formulation. The first one is proportionality, proposed by Steinhaus himself. A division of the cake among $n$ agents is proportional if every agent obtains at least what she considers to be $1 / n$ of the cake. The second one is envy-freeness, proposed by [31] and [29]. A division of the cake is envy-free if no agent prefers the allocation received by any other agent over hers. If the entire cake is allocated, any division that is envy-free is also proportional. Proportionality and envy-freeness are often considered "the two most important tests of equity" [45, p. 166].

The literature has developed procedures that produce envy-free cake divisions when all agents report their preferences over the cake pieces truthfully. We will refer to these as envy-free procedures. Although the ones used for the division of a cake among two agents have been known and used since ancient times, all others rely on complex algorithms which are far from straightforward to general audiences: the Selfridge-Conway procedure for three agents is a case in point. These procedures obtain envy-free allocations at the cost of being hard to understand by the audiences for whom they are intended. If those procedures are not perceived as envy-free in practice (or at least fairer than proportional ones), there would be little support for their implementation in practice. Therefore, the first key question is whether those algorithms produce allocations that are not only fair in theory, but that are also perceived to be fair in practice.

Furthermore, the envy-freeness of these division procedures can only be guaranteed if the agents report their preferences truthfully. If agents strategically misrepresent their preferences, an allocation with envy can be obtained as a Nash equilibrium outcome of the game associated to envy-free procedures [13]. In fact, as we show in Lemma 4, envy can rationally emerge in envy-free procedures even when only one agent lies. Therefore, a second key question is whether cake-cutting procedures are manipulated in practice, and whether such manipulations, if they exist, significantly increase envy. 
Our third and final key question is whether agents can successfully learn their opponents' preferences through repeated interaction. This question is related to the previous two in that an agent needs some information about their opponents' preferences to successfully manipulate a cakecutting procedure. An agent can acquire this valuable information through experimentation, i.e. varying her strategies over time and observing her opponents' best responses to them. If agents do not learn through experimentation, there is little concern about the manipulation of cake-cutting procedures in environments in which agents' preferences are privately known, and thus no concern about the emergence of envy in otherwise envy-free procedures.

We tackle these three questions by means of a lab experiment. In the experiment we study:

(1) the perceived fairness of envy-free and proportional cake-cutting procedures,

(2) the extent to which agents manipulate those procedures, and

(3) whether agents learn their partners' preferences and use that information to their advantage.

We consider the most popular cake-cutting procedures and compare their theoretical properties against their actual performance in the lab. The procedures we consider, which are described in detail in subsection 3.1, are:

- For 2 agents: symmetric and asymmetric cut-and-choose;

- For 3 agents: Knaster-Banach last diminisher, (a discrete adaptation of) Dubins-Spanier moving knife, and Selfridge-Conway;

- For 4 agents: Knaster-Banach last diminisher, (a discrete adaptation of) Dubins-Spanier moving knife, and Even-Paz.

These cake-cutting procedures are well-known in the literature because they all achieve proportional allocations. Furthermore, the asymmetric and symmetric cut-and-choose and the SelfridgeConway procedures are even envy-free. ${ }^{1}$

In the experiment, 131 subjects divide 8 cakes using the aforementioned procedures versus automata agents who play truthfully. Agents divide each cake 7 times in what we call rounds, during which their opponents' preferences remain constant. This gives agents incentives to try to learn their opponents' preferences. In addition, during the final two rounds agents are directly told their opponents' preferences, so that we are able to differentiate between manipulations made to learn the opponents' preferences and those made to directly increase the subjects' immediate payoff. Subjects observe which share of the cake they get in each round and the value (in their own eyes) of their opponents' shares. This way, subjects can assess the fairness of the allocations received. At the end of the experiment, agents complete a questionnaire which asks them to rank the fairness of the division procedures and allows them to give their feedback on their experience with the cake-cutting procedures. Agents are also asked which procedure was the fairest and why.

\subsection{Overview of Results}

We find that all cake-cutting procedures are very frequently manipulated, in fact at least half of the time (subsection 5.4). As a consequence, envy-free procedures generate envy. Envy is generated in $7 \%$ of cases when using the asymmetric cut-and-choose procedure in which the subject cuts the cake, in $18 \%$ of cases when using the symmetric cut-and-choose procedure in which both subjects cut the cake, and in $28 \%$ of cases when using Selfridge-Conway. However, these procedures still generate substantially less envy than their proportional counterparts, which generate envy in over $50 \%$ of cases (subsection 5.3). The difference in envy is noticed by the subjects. Envyfree procedures receive a significantly better fairness ranking than their counterparts (over $80 \%$ of

\footnotetext{
${ }^{1}$ We do not include an envy-free procedure for four agents because the only finite one known to date, proposed by [2], is too complicated to be implemented in the lab.
} 
subjects view them as fair, compared to less than $50 \%$ for their proportional counterparts). Moreover, agents were at least three times more likely to rank envy-free procedures as the fairest ones than proportional ones (subsection 5.1). Overall, the experimental results provide support for the use of the cut-and-choose and Selfridge-Conway procedures, and more generally, of envy-free cake-cutting procedures. These procedures are manipulated in practice just as much as their proportional counterparts, but nonetheless they generate substantially less envy. As a consequence, they are perceived as significantly fairer than their proportional counterparts.

We find some evidence of successful learning, in particular in both of the cut-and-choose procedures, the Knaster-Banach last diminisher, and to some extent in Selfridge-Conway. Surprisingly, we observe that more knowledge does not always yield higher payoffs. This is because agents use that knowledge to manipulate the cake-cutting procedures in the wrong way. In particular, they try to follow simple heuristics that worked in the past, such as cutting the cake a bit more to the right, which may be harmful in other procedures in which the optimal manipulation was to cut the cake a bit more to the left. Overall, we observe that knowledge significantly decreases the level of truth-telling behavior and envy (subsections 5.4 and 5.5). Moreover, we find that about $80 \%$ of the agents do manipulations that are obviously harmful to them, even in the simple cut-and-choose procedure. For example, they cut the cake at a certain location $x$, see that their partner chooses the right piece, and then, at the next play against the same partner, cut to the left of $x-$ which is guaranteed to result in a smaller piece for them (subsection 5.6).

\section{RELATED LITERATURE}

\subsection{Laboratory Experiments}

All fair division experiments that we know of deal with discrete indivisible goods and/or a homogeneous divisible good such as money. This is quite different than our setting, where there is a continuous heterogeneous divisible good. With indivisible goods, the user input usually consists of a ranking of the goods or an assignment of a monetary value to each good. In contrast, cakecutting has a spatial element - the participants have to decide where exactly to cut the cake. Since the user interface, user experience and potential manipulations are different, we cannot automatically expect the findings of previous experiments to hold in our setting too. Keeping this in mind, we survey previous lab experiments and compare their findings with ours.

Sophisticated versus simple. In some experiments, the main research question is which procedure yields more user satisfaction? In particular, do users prefer the allocations generated by sophisticated and provably-fair procedures, to the allocations generated by simple and intuitive procedures?

[54] compare the simple divide-and-choose procedure to the more sophisticated Adjusted-Knaster and Proportional-Knaster procedures, for allocating indivisible goods with monetary compensation. They find that, if the participants truthfully adhere to the protocol, then the sophisticated mechanisms perform better than divide-and-choose in terms of efficiency and fairness. [23] compare five procedures for indivisible object allocation to the allocation with the highest mutual satisfaction scores. They find that the fair division procedures yield allocations that are rather unsatisfactory to humans. They attribute this to temporal fluctuation of human preferences, and non-additivity of valuations. In a different experiment, [24] compare three simple algorithms for allocating indivisible goods to four provably-fair algorithms. They find that, counter-intuitively, the simple algorithms produce significantly fairer allocations.

In contrast, other studies emphasize the advantage of sophisticated fair division procedures. [3] studied division of homogeneous resources using Crawford's negotiation procedures, and found that his procedures induce even selfish players to act fairly. [30] used the spliddit.org website [32] 
to study division of rooms and rent, and found that their maximin procedure performs significantly better than a procedure that selects an arbitrary envy-free allocation. Our findings are in line with the latter studies. Despite the strategic manipulation by humans, the final outcomes of the envyfree procedures (in particular, Selfridge-Conway) are significantly fairer and more satisfactory than the non-envy-free procedures. Thus, at least in our setting, the extra-complexity of the procedures pays back in fairness.

Strategic manipulation. In some experiments, the main goal is to check the strategic behavior of subjects: Do they try to manipulate the protocol? Do they manipulate successfully? And how does the manipulation affect the protocol outcomes? All previous experiments that we know of found that agents do try to manipulate. However, the effect of this manipulation on the outcome depends on the protocol: in simple auction-based protocols, manipulations lead to highly inefficient outcomes, where no deal is done even though a deal is possible $[19,48]$. Using more structured conflict-resolution procedures (such as Adjusted Winner) does not eliminate manipulation, but it does lead to a much more efficient outcome [18, 39].

In our experiment, too, we find that subjects try to manipulate the protocol, and the manipulative behavior increases over time. We also find that some procedures are easier to manipulate than others. In particular, Divide-and-Choose and the Knaster-Banach last diminisher procedure are particularly prone to manipulative behavior. This might be due to their simplicity - procedures that are easier to understand are also easier to manipulate.

Strategic behavior was studied extensively in other markets besides fair division, particularly in matching markets [15]. A remarkable finding in such experiments is that people try to manipulate even when the mechanism is truthful and thus manipulation cannot help [1, 34, 35, 48, 52]. In our experiment this finding is even more pronounced: about $80 \%$ of all subjects tried at least one manipulation that is strictly dominated and obviously results in a smaller payoff for them.

Different desiderata. In some experiments, the main research question is what desiderata are more important to users? Early experiments checked this question in the simple setting of dividing money (a homogeneous resource). Many experiments check whether, in an inherently unfair game such as the ultimatum game, subjects prefer to accept an unfair offer than to accept nothing [33, $40,59]$. Other experiments check whether, when dividing money among others, people prefer a fair inefficient division to an unfair division that is more efficient [25, 27, 36]. It was found that such preferences depend on psychological and cultural factors (e.g. economics students choose differently than law students). Later experiments asked similar questions in more complex settings, involving allocation of indivisible objects [37,38]. These findings are orthogonal to our experiment, in which the fairness desiderata are fixed and the goal is to check which procedure attains them most efficiently.

Besides lab experiments, several fair division procedures were applied to real-life cases, including $[6,11,12,28,41,50,57,57]$. Similarly, computerized simulations of fair division algorithms were used to test properties of such algorithms that are difficult to analyze theoretically, e.g. $[16,21,58]$.

\subsection{Strategic Fair Division}

There are several theoretical studies regarding the strategic properties of cake-cutting protocols $[13,14]$, and various sophisticated protocols that are truthful under some assumptions on the valuations. See [47], [44], [42], [17], [4] and [5]. The repeated-cake-cutting setting has been studied by [20]. They studied ways by which the cutter can exploit her knowledge of the chooser's preferences in order to improve her own welfare. Recently, [56] continued this line of work by suggesting new 
division protocols that are non-exploitable, i.e. a risk-averse cutter cannot improve her welfare using information from previous interactions. Our work complements these theoretic works in that we study the strategies actually used by human subjects when they play repeatedly as cutters.

\section{THEORY}

We consider a standard setup as described in [51]. A cake-cutting problem $\left([0,1], N,\left(v_{i}\right)_{i \in N}\right)$ is a triplet where $[0,1]$ is the cake, $N=\{1, \ldots, n\}$ is the set of agents interested in the cake, and $v_{i}$ is the valuation function of agent $i$, which maps a given subset $I \subseteq[0,1]$ to the value assigned to it by agent $i, v_{i}(I)$. We write $v_{i}(x, y)$ as a shorthand for $v_{i}([x, y])$. We make the standard assumption that $v_{i}$ is a nonatomic measure, i.e., it is an additive set function, it is non-negative, and it assigns a value of zero to subsets of length zero. We normalize the valuation functions so that $v_{i}(0,1)=1$.

We refer to a finite union of disjoint intervals as a piece of cake. An allocation $A$ is a partition of $[0,1]$ into $n$ ordered, pairwise-disjoint pieces of cake $A=\left(A_{1}, \ldots, A_{n}\right)$ such that $A_{1} \cup \ldots \cup A_{n}=$ $[0,1]$. In a non-strategic framework in which all agents reveal their true valuation function, a procedure is a function that takes a cake-cutting problem as input and returns an allocation.

\subsection{Division Procedures}

We consider the following procedures to divide a cake among two agents.

Asymmetric cut-and-choose (2ACC). Agent 1 cuts the cake into two equally-valued pieces, i.e. $t$ wo pieces $\left[0, x_{1}\right)$ and $\left[x_{1}, 1\right]$ such that $v_{1}\left(0, x_{1}\right)=v_{1}\left(x_{1}, 1\right)=1 / 2$. Agent 2 then chooses her preferred piece, and agent 1 receives the remaining piece. Formally, if $v_{2}\left(0, x_{1}\right) \geq v_{2}\left(x_{1}, 1\right)$, then set $A_{2}=\left[0, x_{1}\right), A_{1}=$ $\left[x_{1}, 1\right]$; otherwise set $A_{1}=\left[0, x_{1}\right), A_{2}=\left[x_{1}, 1\right]$.

Symmetric cut-and-choose (2SCC). Both agents cut the cake into two equally-valued pieces by choosing $x_{i}$ such that $v_{i}\left(0, x_{i}\right)=v_{i}\left(x_{i}, 1\right)=1 / 2$. Let agent 1 be the one who chooses the lowest cut point $x_{1} \leq x_{2}$ without loss of generality. Then, agent 1 receives the piece $A_{1}=\left[0, \frac{x_{1}+x_{2}}{2}\right)$, and agent 2 receives the piece $A_{2}=\left[\frac{x_{1}+x_{2}}{2}, 1\right]$.

Both 2ACC and 2SCC have been used and studied since biblical times, yet they are only defined for the division of cake among two agents. Now we define three procedures for dividing cake among three or more players. The first of these is the last diminisher procedure suggested by Knaster and Banach.

Last diminisher for $n$ agents $(n \mathrm{LD})$. Given a cake [y,1], agent 1 chooses a cut $x_{1}$ so that $v_{1}\left(y, x_{1}\right)=$ $v_{1}(y, 1) / n$. Agent 2 now has the right, but is not obliged, to choose $x_{2}<x_{1}$. Whatever she does, agent 3 has the right, without obligation, to further diminish the already diminished (or not diminished) piece too, and so on up to $n$. The rule obliges the last diminisher (say agent $i$ ) who chose the cut $x_{i}$ to take as her allocation $A_{i}=\left[y, x_{i}\right)$. Agent $i$ goes home, and the remaining $n-1$ persons replay the game with the remainder of the cake $\left[x_{i}, 1\right]$. When there is only one agent left, she receives the unclaimed piece.

Similar to $n \mathrm{LD}$ is the moving-knife of [22], in which agents cut the cake simultaneously rather than sequentially. Here we describe a discrete adaptation of it.

Dubins-Spanier for $n$ agents ( $n \mathrm{DS})$. Given a cake [y,1], each agent simultaneously cuts the cake at a point $x_{i}$ such that $v_{i}\left(y, x_{i}\right)=1 / n$. The agent $i^{*}$ who made the leftmost cut exits with the piece $A_{i^{*}}=\left[y, x_{i^{*}}\right]$. Agent $i^{*}$ is disposed of, and the remaining $n-1$ persons start the same game with the remainder of the cake $\left[x_{i^{*}}, 1\right]$. When there is only one agent left, she receives the unclaimed piece.

An alternative procedure was suggested by [26] that improves on $n \mathrm{LD}$ in that it requires fewer cuts to achieve a proportional allocation. The idea of this procedure is to divide the original cake cutting problem into two disjoint ones at each step. 
Even-Paz for $n$ agents ( $n \mathrm{EP})$. For the sake of clarity assume that $n$ is a power of 2. Given a cake $[y, z]$, all agents choose cuts $x_{i}$ such that $v_{i}\left(y, x_{i}\right)=v_{i}(y, z) / 2$. We let $x^{*}$ be the median cut, i.e. the $\lfloor n / 2\rfloor$ th cut. Then the procedure breaks the cake-cutting problem into two: all agents who choose cuts $x_{i} \leq x^{*}$ are to divide the cake $\left[y, x^{*}\right)$, whereas all agents who chose cuts above $x^{*}$ are to divide the cake $\left[x^{*}, z\right]$. Each half is divided recursively among the $n / 2$ partners assigned to it. When the procedure is called with a singleton set of agents $\{i\}$ and an interval $I$ it assigns $A_{i}=I$.

The last three procedures $n \mathrm{DS}, n \mathrm{LD}$ and $n \mathrm{EP}$ can be adapted to divide a cake among any number of agents. Our last procedure is only suitable for dividing cake among 3 agents. It differs from the previous procedures in that it generates allocations that are not contiguous. Furthermore, it requires not one but two cake cuts to be made at the same time.

Selfridge-Conway (3SC). Agent 1 cuts the cake into three pieces of equal value to her: $I_{1}, I_{2}, I_{3}$; so that $v_{1}\left(I_{i}\right)=1 / 3$. Agent 2 divides the piece of highest value to her, say $I_{1}$ into $I_{1}^{\prime}$ and $T=I_{1} \backslash I_{1}^{\prime}$, so that the value of $I_{1}^{\prime}$ is the same of the second most valuable piece, say $I_{2}: v_{2}\left(I_{1}^{\prime}\right)=v_{2}\left(I_{2}\right)$. We separate the original cake into the modified cake $C^{\prime}=C \backslash T$ and the trimmings $T$. First we allocate $C^{\prime}$. Let agent 3 choose and take her favorite piece among $I_{1}^{\prime}, I_{2}, I_{3}$. If she chooses $I_{1}^{\prime}$, let agent 2 choose any remaining piece; but if agent 3 chooses $I_{2}$ or $I_{3}$, then give $I_{1}^{\prime}$ to agent 2 without letting her choose. Agent 1 receives the leftover piece. Now we assign $T$. Let $i \in\{2,3\}$ be the player who obtained $I_{1}^{\prime}$, and $j$ the other one Agent $j$ splits $T$ into three parts of equal value to her. Now agent $i, 1$, and $j$ choose a piece of $T$ in that specified order.

\subsection{Fairness Properties}

We consider the following fairness properties of allocations. An allocation $A$ is proportional if each agent gets at least $1 / n$ of the cake according to her own evaluation, i.e. if $\forall i \in N: v_{i}\left(A_{i}\right) \geq 1 / n$. An allocation $A$ is envy-free if no agent prefers another agent's share, i.e. $\forall i, j \in N: v_{i}\left(A_{i}\right) \geq v_{i}\left(A_{j}\right){ }^{2}$

In our setup, envy-freeness implies proportionality, while the converse is true only for the case of two agents. A procedure is envy-free or proportional if, for every cake-cutting problem, it produces an allocation that is envy-free or proportional, respectively. The following lemma summarizes the well-known fairness properties of these procedures, and thus is stated without proof (see [11, 53]).

Lemma 1. 2ACC, $2 S C C, n D S, n L D, n E P$ and $3 S C$ are all proportional. $2 A C C, 2 S C C$, and $3 S C$ are envy-free. $n D S, n L D$, and $n E P$ are not envy-free.

The previous lemma gives us our first hypothesis. Namely, when dividing a cake among 3 agents,

Hypothesis 1. Agents perceive the allocations received under $3 \mathrm{SC}$ as fairer than those received under 3DS and 3LD.

This is an important hypothesis to test because it is evident that the envy-freeness of 3SC comes at the cost of being considerably more complex than 3DS or 3LD. Therefore, if 3SC is not considered a fairer option than 3DS and 3LD, then there will be little support for its use in real-life applications.

\subsection{Incentive Properties}

Another important goal of cake-cutting procedures is to give incentives to agents to reveal their true (privately known) valuation function to a mediator who, after receiving the report from all agents, conducts a division procedure. The valuation function is partially revealed via a series of cake cuts or choices between pieces of cake. Although the mediator does not know the valuations, we assume that every agent knows the other agents' valuations.

\footnotetext{
${ }^{2}$ This notion should not be confused with procedural envy-freeness or anonymity [47].
} 
In a strategic framework, given a cake $[0,1]$ and a set of agents $N$, a procedure $p$ is a function from the revealed valuation function of each agent to an allocation $A$. We write $p_{i}\left(v_{i}, v_{-i}\right)=A_{i}$ to denote the cake allocated to agent $i$ by procedure $p$, where $v_{-i}$ denotes the reported valuation functions of all other agents except $i$. The procedure $p$ is strategy-proof if for every agent $i$, every collection of valuation functions $\left(v_{i}, v_{-i}\right)$, and every other valuation function $v_{i}^{\prime}, v_{i}\left(p_{i}\left(v_{i}, v_{-i}\right)\right) \geq$ $v_{i}\left(p_{i}\left(v_{i}^{\prime}, v_{-i}\right)\right)$. Note that the definition is a strict one: a procedure is strategy-proof only if behaving truthfully is a dominant strategy for every player. $^{3}$ Therefore, it is not surprising that:

Lemma 2. 2ACC, $2 S C C, n D S, n L D, n E P$ and $3 S C$ are all not strategy-proof.

Lemma 2 is also well-known; [7] in particular discuss many examples of how all these procedures can be manipulated. We may ask how much value agents can gain by strategic behavior compared to their guaranteed payoff obtained by truth-telling in any of the procedures we have described. We answer this question by considering the notion of $\epsilon$-strategy-proofness, which has recently been suggested in the literature [43]. In layman terms, a cake-cutting procedure is $\epsilon$ strategy-proof if there is no cake-cutting problem for which a misrepresentation of preferences guarantees more than $\epsilon$ utility compared to truth-telling. ${ }^{4}$ Ideally, a proportional procedure should have an $\epsilon=0$, and in the worst case a procedure would have $\epsilon=\frac{n-1}{n}$ : this means that truthful behavior guarantees an agent $\frac{1}{n}$, whereas lying yields the maximum utility possible (1). Unfortunately, we show that all the procedures we consider can offer the largest incentives for preference manipulation.

Lemma 3. The procedures $2 A C C, 2 S C C, n D S, n L D, n E P, 3 S C$ are $\frac{n-1}{n}$ strategy-proof and this is tight.

We postpone the constructive proof of Lemma 3 to the Appendix. The previous two lemmata suggest that if agents know their opponents' preferences, they should not behave truthfully if the cake-cutting problem admits a successful manipulation. This is our second hypothesis.

Hypothesis 2. Agents who know their opponents' preferences do not behave truthfully in 2ACC, 2SCC, $n \mathrm{DS}, n \mathrm{LD}, n \mathrm{EP}$ and 3SC.

The assumption that agents know their partners' valuations is a strong one, yet necessary for agents to manipulate the procedure to their advantage with certainty of success. Without such knowledge, an agent might perform a manipulation that will decrease its utility. Therefore, in the fair division literature, it is often claimed that strategic manipulation is not an issue when people do not know their partners' preferences (see e.g. [30]). However, in real life, agents may have a partial knowledge about their partners' preferences, particularly if they have interacted with those partners previously. In those cases, an agent is able to learn the other agents' valuations through experimentation, i.e. choosing different strategies each interaction in order to eventually improve their own allocation. This simple observation provides us with our final hypothesis.

Hypothesis 3. Agents who do not know their opponents' preferences but who repeatedly interact with them, successfully learn their opponents' preferences and do not behave truthfully in 2ACC, 2SCC, 3SC, $n \mathrm{DS}, n \mathrm{LD}, n \mathrm{EP}$ and 3SC.

\subsection{Fairness and Incentives}

It is important to note a dependency between our three hypotheses. Hypothesis 1 states that 3SC is perceived as fairer than 3LD and 3DS since it generates envy-free allocations. However, this

\footnotetext{
${ }^{3}$ This is the standard notion of strategy-proofness in mechanism design. For a weaker notion see $[7,8]$.

${ }^{4}$ Formally, for any $\epsilon \in[0,1]$, the procedure $p$ is $\epsilon$-strategy-proof if for every agent $i$, every collection of valuations functions $\left(v_{i}, v_{-i}\right)$, and every other valuation function $v_{i}^{\prime}, v_{i}\left(p_{i}\left(v_{i}, v_{-i}\right)\right) \geq v_{i}\left(p_{i}\left(v_{i}^{\prime}, v_{-i}\right)\right)-\epsilon$.
} 
envy-freeness is guaranteed only when all agents report their preferences truthfully. In contrast, Hypotheses 2 and 3 state that people do not behave truthfully. If all agents do not behave truthfully, then in general, all three procedures discussed - 3SC, 3LD and 3DS - generate envy [13]. However, hypothesis 1 still holds if the procedures are used by a population in which a fraction $\alpha$ of agents behave truthfully. Then 3SC guarantees envy-freeness in at least $\alpha$ cases, and thus it is reasonable to expect that it would still be perceived as fairer than 3LD and 3DS. As a consequence, Hypothesis 1 extends to cases in which a constant fraction of the agents behave truthfully. ${ }^{5}$

A related interesting question is whether envy can be generated in 3SC when only one agent misreports her preferences, while the other agents are truthful. This question is particularly relevant to our experimental setting, since in our setting the computerized agents are truthful so only the single human subject might act strategically. ${ }^{6}$ We answer this question in the affirmative in our following lemma (we postpone the proof to the Appendix).

Lemma 4. Envy can be generated in $3 S C$ with just one agent misrepresenting her preferences. This agent achieves a higher payoff at the cost of being envious.

\section{EXPERIMENT}

\subsection{Design}

The subject divides a cake with 1,2, or 3 other agents. The other agents are automated; they are programmed to cut the cake truthfully. Fixing the behavior of the other agents in this fashion is necessary in order to test our hypotheses. The subject divides one cake using each of the division procedures we have described. The subject is not told that the other agents are automata that behave truthfully.

The cake-cutting occurs in the following order: 2ACC, 2SCC, 3DS, 4DS, 3LD, 4LD, 4EP, and 3SC. That is, the subjects divide 8 cakes. This particular order was chosen so that subjects solve the easiest problems first. In the first 7 procedures the subjects need to move just one knife (in 3SC subjects move two), and same procedures in which just the number of subjects varies (like 3DS-4DS and 3LD-4LD) are played consecutively to make it easier for the subjects.

We change the names of the procedures to make it easier for the subjects to understand them. We use the following names: I Cut You Choose (for ACC), Cut Middle (for SCC), Leftmost Leaves (for $n \mathrm{DS}$ ), Last Challenger (for $n \mathrm{LD}$ ), Super Fast (for 4EP) and Super Fair (for 3SC). We choose the name Super Fair to emphasize that this procedure is mathematically designed to produce a fair allocation. Admittedly, this biases the subjects to rank this procedure higher in a scale of fairness. Yet, whenever scientists have applied allocation mechanisms in practice, they explicitly emphasize that one can show that such procedures are fairer. ${ }^{7}$ Thus, it is likely that the same approach would be used if the Selfridge-Conway procedure was to be applied in practice. As we will see, 3SC is highly ranked with regard to fairness because subjects realize that it generates envy-free allocations in most cases, so there is little concern that our findings with respect to fairness are solely due to the chosen name.

Each cake is divided 7 times. We call each of these divisions a round. During the first five rounds, the subject does not know the other agents' valuations. In the remaining two rounds, the subject

\footnotetext{
${ }^{5}$ The fraction $\alpha$ is in fact not constant but specific to each procedure. However, from our lab experiment we found that the fraction of agents who behave truthfully in 3SC (45\%) is larger than in 3DS (40\%) and 3LD (16\%), and thus it is safe to expect that $3 \mathrm{SC}$ is indeed perceived fairer than 3DS and 3LD.

${ }^{6}$ Brânzei et al. 13 proved that envy can be generated in Nash equilibrium of 3SC, but their proof crucially relies on the assumption that all three agents behave untruthfully.

${ }^{7}$ For example, the introduction of the deferred acceptance mechanism in Boston was accompanied by a campaign informing the public that the new allocation method was non-manipulable and in a sense fairer [49]. Similarly, the fair division website Spliddit.com tells its users that its division methods achieve specific fairness guarantees such as envy-freeness.
} 
observes the automata's valuations. We give subjects 5 rounds to experiment and learn their opponents' valuations. The valuations of the subject and the automata are constant during the 7 rounds in each procedure, but they change once the agents move to the next procedure. The subject makes the first cut in all procedures that are sequential.

In all procedures, the cake is a line and the subjects' and automated agents' valuations are normalized so that $v_{i}(0,1)=120$. In other words, the subject and the automata can obtain a maximum of 120 points if they obtain all of their desired parts of the cake. We chose 120 because it is easily divisible by 2,3 , and 4 . Subjects are shown their valuations on the computer screen. Their valuations are given by a set of subintervals which are deemed desirable, and another set which are not (described in the Appendix). All desired intervals of the same length yield the same payoff; such valuations are known as piecewise uniform. The cake can only be cut in a position $x$ so that $v_{i}(0, x)$ equals an integer number between 0 and 120. A representative screen is shown in Figure 1. The graphical interface can be downloaded here.

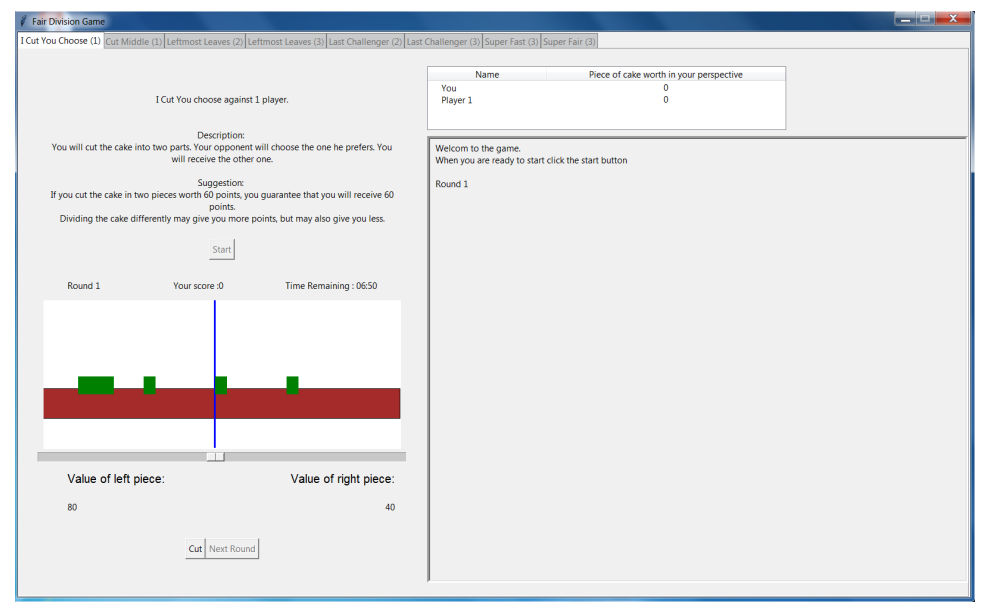

Fig. 1. An example of our experimental interface. The cake is depicted as a brown line, while the desirable parts of the subject are emphasized with a green color.

Each subject has 7 minutes to complete the 7 rounds of each procedure. If they do not take any action after the time is over they receive 0 points for the remaining rounds. The clock pauses each time the subject moves to the next round, and she selects when to start each round. The time constraint was almost never binding in the real experiment. After completing each round, subjects are told (in the top right corner of the graphical interface) what share of cake they got, and the valuation of the shares that the automated players received, calculated by the valuation function of the subjects, so that the subjects can properly assess the fairness of the division procedure.

We choose the valuations so that strategic behavior yields substantial benefits over truthful behavior. The valuations are described in the Appendix. The subjects are given the suggestion to cut the cake truthfully, but are also explicitly told that they can choose another strategy that may give them more or less points than the truthful one.

After cutting all the cakes, subjects are asked to rank the perceived fairness of the procedures on a scale from 1 (very fair) to 4 (very unfair). ${ }^{8}$ Subjects are also asked which of all the procedures

\footnotetext{
${ }^{8}$ Subjects are only asked to provide a ranking for 2ACC, 2 SCC, $n \mathrm{LD}$ and $n \mathrm{DS}$. This ranking was not asked for 3SC and 4EP; however, the fairness of these procedures can be assessed via other questions, as we will discuss. The Appendix describes all the questions asked.
} 
was the fairest in their opinion and why. We also ask them if they would have preferred to bargain directly with their opponents in a decentralized fashion. Subjects also give their comments freely in a textbox.

At the end of the experiment, 2 rounds are randomly selected from all procedures, and subjects are paid the number of points they obtained in both procedures divided by 10, in GBP. In addition, subjects receive a fixed 5 GBP payment for showing up. For example, if in the two randomly selected rounds the subject obtains 120 and 80 points, then she receives $12+8+5=25 \mathrm{GBP}$. The highest payment achievable (through strategic behavior) is $29 \mathrm{GBP}$, whereas the lowest is $5 \mathrm{GBP}$.

\subsection{Implementation}

The experiment was conducted at the lab facilities at the corresponding University. The experiment took place during over the course of seven sessions on July 5, 6, 12 and 13 of 2018. Upon their arrival to the lab, subjects were randomly assigned to a computer. They signed a consent form and were given the experiment instructions in a short presentation by the principal investigator (these are provided in the Appendix). They were allowed to ask questions during and after the instructions were given. After all questions had been answered, the subjects were allowed to start the experiment. Subjects were not allowed to communicate with anyone during the experiment. After the experiment ended, subjects were paid in private and dismissed.

We had 136 participants. Unfortunately, 5 of the responses for the fairness questionnaire could not be processed due to technical issues, so our total sample for this part is only 131. Similarly, due to technical problems we lost the observations of the actual cake cuts performed by 3 subjects, so our total sample for this part is 133 . The average payment was $\$ 14$. The highest payment was $\$ 28$ and the lowest was $\$ 5$. The distribution of payments is shown in Figure 7 in the Appendix. On average, subjects took around 45 minutes to complete the experiment. The data is available from the University data repository and here and here.

\section{RESULTS}

\subsection{Fairness}

We find evidence suggesting that envy-free procedures are considered fairer than proportional ones. This evidence comes from two observations. First, when the subjects were asked specifically to rank each procedure on the very fair to very unfair scale, 2ACC and 2SCC were considered fairer than $n \mathrm{DS}$ and $n \mathrm{LD}$. Whereas over $80 \%$ of our subjects considered the former two to be fair or very fair ( $82 \%$ and $88 \%$ respectively), less than half considered the latter two as fair or very fair (only $47 \%$ and $43 \%$ ). A Wilcoxon signed-rank test indicates that the fairness ranking of the first group of procedures (2ACC and 2SCC) is statistically different $(z=-9.332, p<0.001)$ than the one of the second group of procedures ( $n \mathrm{DS}$ and $n \mathrm{LD}$ ). Table 1 further describes these statistics.

Second, at the end of the experiment, when subjects stated their opinions about which procedures produced the fairest allocations (they could list more than one), 53 listed 2ACC, 37 listed 2SCC, and 36 listed 3SC. In contrast, only 12,11 , and 4 chose $4 \mathrm{EP}, n \mathrm{DS}$, and $n \mathrm{LD}$, respectively. The corresponding graph appears in Figure 2. It is clear that the fact that the first three produced envy-free allocations influenced their choices. For example, one subject wrote: "the fairest of all the games was probably super fair, from memory there was little deviation (sic) the results between all the players". Another subject explained: "I think the one with the two sliders was the fairest. Everyone received the same amount, at least in my scenario as they were all divided equally". 9 Thus, we conclude the following.

\footnotetext{
${ }^{9}$ This comment is also related to a different fairness notion in the literature called equitability. This property is at odds with envy-freeness if Pareto optimality is imposed [10].
} 
Table 1. Subjects' views on fairness (131 subjects).

\begin{tabular}{ccccc}
\hline \hline & Very Fair & Fair & Unfair & Very Unfair \\
\hline 2ACC & 17 & 90 & 22 & 2 \\
& $(13 \%)$ & $(69 \%)$ & $(17 \%)$ & $(2 \%)$ \\
2 SCC & 11 & 104 & 14 & 2 \\
& $(8 \%)$ & $(79 \%)$ & $(11 \%)$ & $(2 \%)$ \\
$n \mathrm{LD}$ & 4 & 58 & 63 & 6 \\
& $(3 \%)$ & $(44 \%)$ & $(48 \%)$ & $(5 \%)$ \\
$n \mathrm{DS}$ & 4 & 52 & 57 & 16 \\
& $(3 \%)$ & $(40 \%)$ & $(44 \%)$ & $(12 \%)$ \\
\hline \hline
\end{tabular}

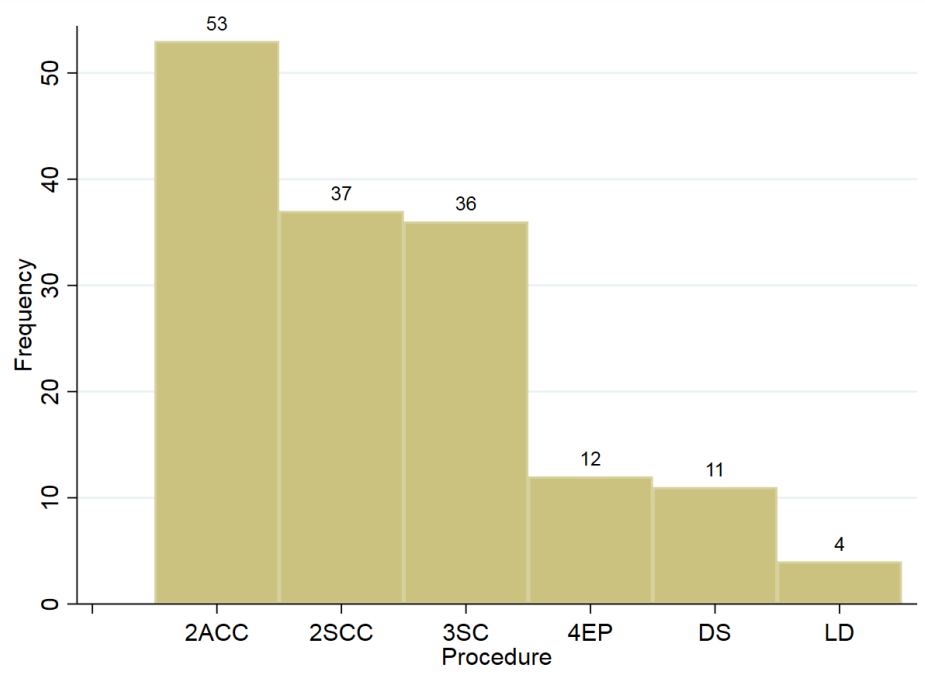

Fig. 2. Which is the fairest procedure in practice?

Result 1. Subjects perceive $2 A C C, 2 S C C$, and $3 S C$ (all envy-free procedures) as fairer than $n L D, n D S$, and $n E P$ (all proportional procedures).

We also find that a higher fraction of our subjects (56\% versus $44 \%$ ) said that they found $3 \mathrm{SC}$ fairer than all other procedures (including 2ACC and 2SCC) when explicitly asked so, although we cannot guarantee that such answering patterns differ from random choice (binomial one-sided test $\mathrm{p}$-value $=0.11)^{10}$

\subsection{Procedure versus bargaining}

We asked subjects whether they would have preferred to directly bargain with the other agents instead of participating in a centralized procedure. Since decentralized bargaining over the cake is always an option, a centralized procedure should represent a guaranteed improvement on this basic alternative. Surprisingly, most subjects said that they would have preferred to bargain in a

\footnotetext{
${ }^{10}$ We also asked them if they found super fast to be any easier than all other procedures. Most subjects (57\% versus $43 \%$ ) said no. We cannot assure that this difference is statistically different from random choice (binomial one-sided test $\mathrm{p}$-value $=0.06$ ).
} 
decentralized fashion (48\%), compared to $29 \%$ who preferred some of the procedures in the experiment (the remaining $23 \%$ were indifferent). Subjects seemed to think that bargaining would improve the position of the agent receiving the worst piece of cake. One subject wrote: "Sometimes. In some cases you could probably bargain and say: look, you clearly got the best deal out of all of these, let me at least have one big catch".

Subjects emphasized the importance of common knowledge of preferences in the decentralized bargaining option: "If we could all see each others chocolate or coconut pieces then a direct negotiation would have been easier to ensure fairness between parties. If all involved where still in the dark about the opponents' chocolate/coconut direct bargaining may not have made a difference". Another subject wrote: "At least we get to know what everybody wants and we can discuss to make the fairest share".

These results were quite unexpected, so our experimental system was not able to explore them out in more depth. As future work, we plan to conduct an experiment for explicitly checking the fairness and efficiency of various forms of decentralized bargaining in fair division.

\subsection{Envy}

Envy emerges in all of the division procedures, although at quite different rates. We define envy as whether a subject prefers, in her own view, the piece of cake received by another subject (even by one point; a robustness analysis is carried out in the Appendix). The percentage of cases in which envy emerges in each procedure is summarized in Table 2.

Table 2. Percentage of cases where envy is generated, by round and on average

\begin{tabular}{llllll|l|ll|l|l}
\hline \hline $\begin{array}{c}\text { Round } \\
\text { Proc }\end{array}$ & 1 & 2 & 3 & 4 & 5 & $\begin{array}{l}\text { No knowl. } \\
\text { average }\end{array}$ & 6 & 7 & $\begin{array}{l}\text { Knowl. } \\
\text { average }\end{array}$ & $\begin{array}{l}\text { Total } \\
\text { average }\end{array}$ \\
\hline 2ACC & 10 & 8 & 8 & 5 & 8 & 8 & 5 & 3 & 4 & 7 \\
2SCC & 16 & 25 & 23 & 20 & 13 & 19 & 17 & 14 & 16 & 18 \\
3DS & 57 & 68 & 62 & 62 & 59 & 62 & 74 & 68 & 71 & 64 \\
3LD & 56 & 53 & 51 & 53 & 43 & 51 & 44 & 40 & 42 & 49 \\
3SC & 31 & 31 & 29 & 29 & 32 & 30 & 23 & 25 & 24 & 29 \\
4DS & 64 & 86 & 84 & 78 & 77 & 78 & 78 & 73 & 76 & 77 \\
4LD & 62 & 66 & 53 & 54 & 50 & 57 & 53 & 44 & 49 & 55 \\
4EP & 97 & 92 & 94 & 93 & 92 & 94 & 91 & 89 & 90 & 93 \\
\hline \hline
\end{tabular}

Envy may emerge in envy-free procedures due to two reasons. One is that subjects strategically manipulate their cake cuts. Another is that subjects did not understand the procedure. For example, in $2 \mathrm{ACC}$, the simplest of the procedures, envy was generated in $3 \%$ of the cases in the last round when subjects knew their opponents' preferences and were already familiar with the division procedure. In these cases, envy was generated by mindless cake cuts. The data for $2 \mathrm{ACC}$ suggest that this dull behavior rarely occurs. Most of the envy is instead caused by strategic experimentation of the subjects, and reduces once subjects know their opponents' preferences in 2ACC and 2SCC. It is somewhat surprising that envy is generated in $2 \mathrm{SCC}$, which we observe is due to the fact that subjects follow the simple heuristic of copying a manipulation strategy that was successful in the past (cut a bit further to the right of the truthful cut).

With the exception of 3SC, envy is generated in half or more of the cake cuts in all procedures for 3 and 4 agents. In particular, envy is generated in over $90 \%$ of the cases when 4EP is used. This finding is intriguing because $4 \mathrm{EP}$ was known to perform well with regards to envy in that it minimizes the maximum number of players who can be envied among all proportional procedures 
[9]. It is interesting that subjects realize that proportional procedures produce envy and that this observation is taken into account when they rank the fairness of the allocations generated.

Overall, envy decreases after subjects learn their opponents' preferences. To see this, we conduct a logit regression for repeated measures of the probability of the emergence of envy on the round number. The associated coefficient is -0.04 and is statistically significant ( $p$-value $<0.001$ ). When we look at each procedure specifically, we observe that envy increases with more knowledge only for $n \mathrm{DS}$ (for 3DS it is statistically significant, but for 4DS it is not).

We summarize these findings as follows.

Result 2. 2ACC, $2 S C C$, and $3 S C$ (all envy-free procedures) generate substantially less envy than $n L D$, $n D S$, and $n E P$ (all proportional procedures). Overall envy decreases over time except in $n D S$.

\subsection{Manipulation}

The findings with respect to manipulation and learning can be previewed in Figure 3, which presents the average number of points obtained in each round, by procedure.

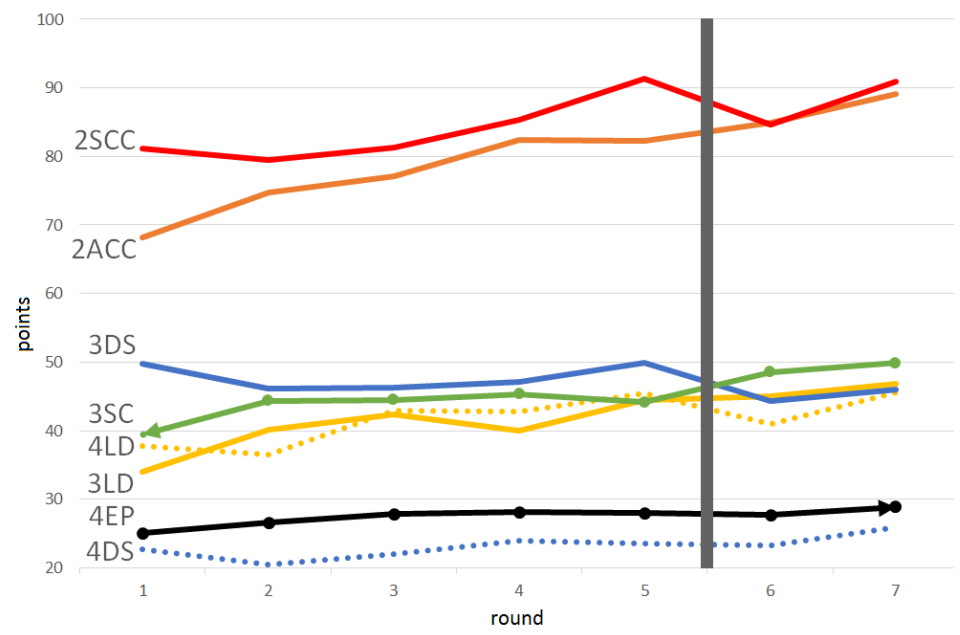

Fig. 3. Average points obtained by the subjects in each round, by procedure.

Figure 3 makes evident that subjects manipulate the procedures, even before they have any information about their opponents' preferences. The average number of points obtained in the first round of $2 \mathrm{ACC}$ is 68 , higher than those obtained with a truthful report (60). Similarly, the average number of points in the first round of 3DS is 50,10 points above those guaranteed with a truthful report. However, the manipulation at such an early stage of the game also has negative consequences: in the first round of 2SCC, the average number of points obtained was 81,9 points below the payoff with truthful behavior. Similarly, in the first round of 4DS, the subjects obtain an average payoff of 23 points, 7 below those guaranteed by truthful behavior.

Figure 4 presents the percentage of payoffs generated by truthful reports (we define a truthful report as one generating a payoff within \pm 5 points of the one corresponding to the suggestion given by the procedure; a robustness analysis is carried out in the Appendix). We focus on payoffs and not on the exact reports, which implies that we only analyze manipulations which affect the 
subjects' payoffs. Those that do not are inconsequential and thus we ignore them in the main text (but discuss them in detail in the Appendix). ${ }^{11}$
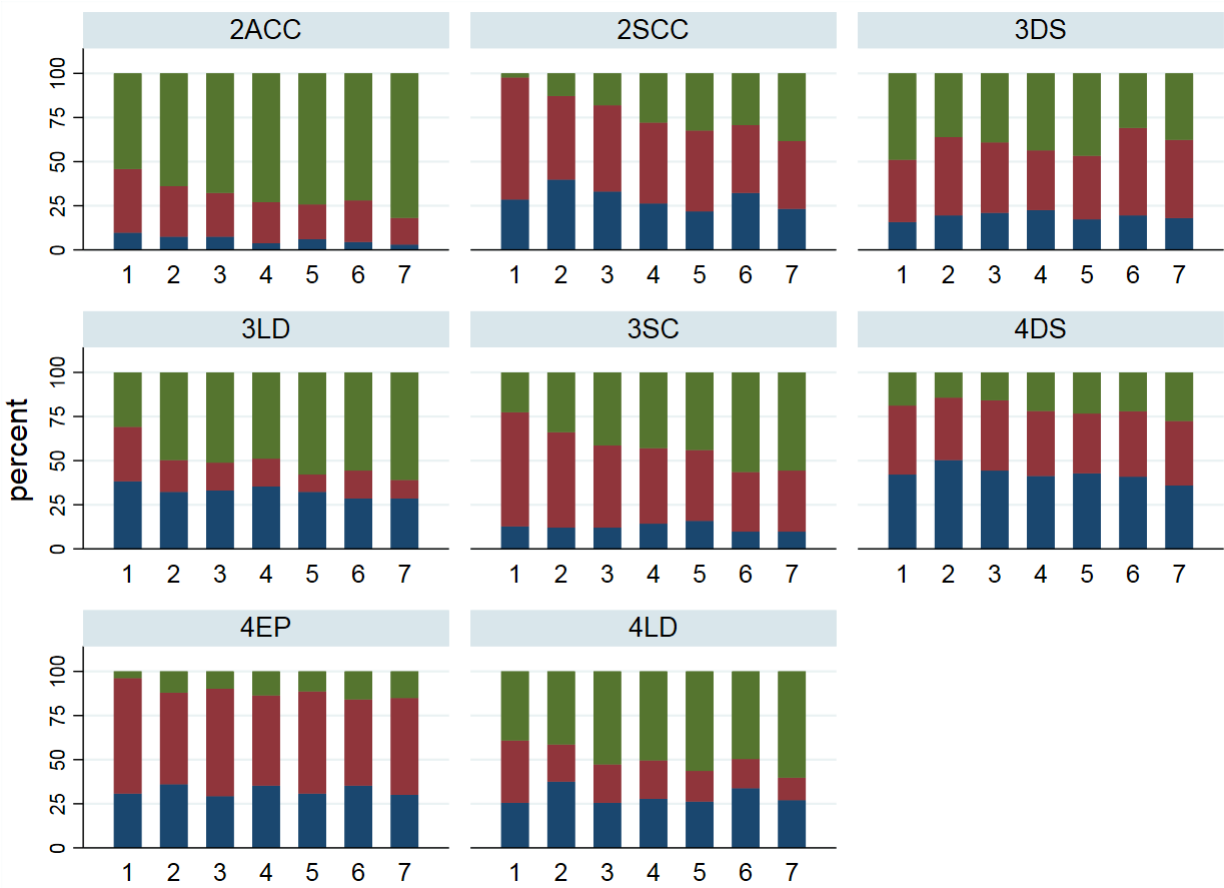

Fig. 4. Percentage of truth-telling (red) and successful (green) and unsuccessful (blue) manipulations over rounds, by procedure. Manipulations are defined as deviations over 5 points from truth-telling.

Figure 4 shows that, in the first round, truth-telling exceeds strategic behavior in only three procedures: 2SCC (69\%), 3SC (65\%), and 4EP (65\%). However, this percentage of truth-telling behavior quickly diminishes as subjects learn their opponents' preferences, as we will see in the next subsection. Similarly, in rounds 6 and 7, when subjects are told their opponents' preferences directly, the percentage of truth-telling diminishes as well: it is only above $50 \%$ for $4 \mathrm{EP}(52 \%)$. This finding suggests that subjects find it harder to manipulate procedures that are more complicated such as $4 \mathrm{EP}$. Subjects successfully manipulate procedures as complicated as 3SC, an observation we did not anticipate: over 55\% of the subjects in rounds 6 and 7 successfully manipulated 3SC.

It is also evident that the percentage of truth-telling behavior becomes smaller in later rounds. To observe this, we conduct a logit regression for repeated measures of the probability of playing truthful strategies on the round number. The obtained coefficient is -0.1 (note the negative sign) and is statistically significant ( $\mathrm{p}$-value $<0.001$ ). Thus, we conclude that:

Result 3. Subjects manipulate (often unsuccessfully, and even without knowing their opponents' preferences) all the division procedures, namely $2 A C C, 2 S C C, n L D, n D S, 4 E P$, and 3SC. Truth-telling decreases over time.

\footnotetext{
${ }^{11}$ Because all other agents behave truthfully, it means that if we observe a payoff different to the one guaranteed by truthtelling, we can assure that the subject misreported her preferences. Thus, the numbers reported are a lower bound on the total number of manipulations, which only reinforces our point that procedures are very frequently manipulated.
} 
The previous result is in line with the findings of [39] who, in a different fair division procedure, in which two subjects vote for a series of issues, also document truthful behavior decreasing over time. We also analyze the manipulation of the cake-cutting procedures by looking at the actual position of the cake cuts made by subjects. The results are very similar and thus postponed to the Appendix.

\subsection{Learning}

Does knowledge help? Knowing the opponents' preferences helped the subjects to earn more points only in 2ACC, $2 \mathrm{SCC}$, 3SC, and 3LD (we compare the number of points obtained in rounds 1-5 versus those obtained in rounds 6-7, see Table 14 in the Appendix). In the case of 3DS, knowledge actually hurts the subjects, although the payoff difference is not statistically significant.

We find that most of the benefits of knowledge come from learning via experimentation. Table 15 in the Appendix shows that the payoffs obtained in round 5 compared to those in round 1 are significantly higher in $2 \mathrm{ACC}, 2 \mathrm{SCC}, n \mathrm{LD}$ and $3 \mathrm{SC}$. In comparison, revealing the opponents' preferences directly (round 7) only affects the payoffs obtained with the knowledge of experimentation (round 5) in 2ACC and 3SC, as shown in Table 16 in the Appendix.

To further asses the impact of learning by experimentation in the subjects' payoffs, we conduct a regression for repeated measures of the scores on the round number up to round 5 . We find that the associated coefficient is 1.6 and is statistically significant (p-value $<0.001$ ). This confirms our finding that subjects can figure out their opponents' preferences via experimentation.

\subsection{Quality of Learning}

How good are people at learning the other agent's valuations? To investigate this question we present a model of rational learning in 2ACC. We consider $2 \mathrm{ACC}$ played for $T$ rounds, where in each round, Alice cuts the cake and Bob chooses a piece. Our model follows the experiment setup, particularly: i) the game is discretized: the cake is $[0, c]$ for some integer $c$ (in the experiment $c=600$ is the number of pixels in the cake); Alice may cut only in integer locations; a cut in $x$ means that the left piece is $[0, x]$ and the right piece is $[x+1, c]$, and ii) Bob is deterministic and non-strategic: he always picks the most valuable piece for him, and if the pieces have equal value, he breaks the tie by selecting the left piece.

Alice's payoff depends only on Bob's half point - the integer $h$ for which $v_{B}(0, h)=v_{B}(h+1, c)=$ $v_{B}(0, c) / 2$. If Alice cuts at some $x<h$, then Bob takes the right piece and she gets $[0, x]$; if Alice cuts at $x \geq h$, then Bob takes the left piece and she gets $[x+1, c]$.

If Alice knows $h$, then it is optimal for her to cut either at $h-1$ or at $h$; in the former case she gets $[0, h-1]$ and in the latter case she gets $[h+1, c]$. Therefore Alice can guarantee a utility of:

$$
u^{o p t}(h)=\max \left[v_{A}(0, h-1), v_{A}(h+1, c)\right]
$$

Initially, Alice does not know $h$, but she can learn the possible range of $h$ from Bob's choices: if Alice cuts at some $s \in[0, c]$ and Bob chooses the right piece, she learns that $h>s$; similarly, if Alice cuts at $t \in[0, c]$ and Bob chooses the left piece, Alice learns that $h \leq t$. In each round, Alice's knowledge about Bob is summarized by two numbers $s<t$ that represent the lower and upper bounds for Bob's half-point $h$, i.e., $s<h \leq t$. With this knowledge, cutting at any $x<s$ is dominated by cutting at $x=s$ (since in both cases Alice will get $[0, x]$ ), and cutting at any $x>t$ is dominated by cutting at $x=t$ (since in both cases Alice will get $[x+1, c]$ ). We say that Alice is rational if all her cuts (from the second round onwards) are undominated.

In 2SCC a similar analysis is applicable. Alice does not know the exact location of Bob's cut (as in our experiment, the subjects were not shown the cut-location of their opponents). Therefore, the only information she can use for learning is whether she receives the leftmost or the rightmost 


\begin{tabular}{|c|c|c|}
\hline & Undominated actions in rounds $2-7$ & Rational players \\
\hline 2ACC & $487 / 795=61.3 \%$ & $32 / 133=24.1 \%$ \\
\hline 2SCC & $447 / 795=56.2 \%$ & $23 / 133=17.3 \%$ \\
\hline
\end{tabular}

Table 3. Number of undominated strategies and rational subjects in the two-agent procedures.

piece. This is analogous to the information she has in 2ACC, so she can learn the possible range for $h$ in a similar manner.

Our findings regarding rational agents and the use of undominated strategies in the experiment are summarized in Table 3. Interestingly, in both games, only about $60 \%$ of all cuts are undominated, and only about $20 \%$ of all players are fully rational (i.e. all their actions are undominated). While this finding is in line with previous studies showing that human subjects often play dominated strategies $[1,34,35,48,52]$, we find it somewhat surprising that so many people behave in a way that is so clearly irrational.

\subsection{Time}

Our software recorded the exact time at which each cake cut was made. This allows us to compare the time that subjects spent on each procedure, which we use as a proxy of the perceived complexity of the procedure. This proxy is noisy, as the order in which subjects use the procedures is fixed, and thus procedures that appear first may take longer than those that appear later simply because the subjects take some time to get used to the experiment interface. Another source of noise is that the time is counted from the time subjects make the cake cut in the first round until they made the last one in round seven, and thus does not count the time that subjects spend reading the instructions of each procedure before they click start. Nevertheless, we believe this data is still informative, and thus we present an overview of it in Figure 8 in the Appendix.

As expected, the first procedure that subjects face (2ACC) is the one in which they spend most time. However, it is interesting that for 2ACC, $n \mathrm{DS}$ and $4 \mathrm{EP}$ subjects spend almost twice as much time as for $n \mathrm{LD}, 2 \mathrm{SCC}$, and 3SC. We do not observe that the procedures that appear later in the experiment take less time to be completed overall. It is intriguing that, even though $n \mathrm{LD}$ and $n \mathrm{DS}$ are theoretically equivalent, subjects spent more time on $n \mathrm{DS}$ than on $n \mathrm{LD}$. This finding is robust (it occurs in both the 3 and 4 agents case) and significant (the corresponding p-value for a t-test testing the null hypothesis that the difference in time spent in both procedures is zero is 0.000 in both cases). Given that $n \mathrm{LD}$ also generates less envy than $n \mathrm{DS}$ in both the 3 and 4 agents case, our findings suggest that overall $n \mathrm{LD}$ performs better in practice than $n \mathrm{DS}$. We could not find a convincing explanation to this difference.

\section{CONCLUSION AND FUTURE WORK}

The envy-freeness of the Selfridge-Conway procedure can only be guaranteed when agents do not manipulate the cake-cutting procedures. In the lab, we find that this is a real concern, as subjects very often report their preferences untruthfully. Nevertheless, the Selfridge-Conway procedure is perceived as significantly fairer than other proportional procedures. Furthermore, it generates substantially less envy in objective terms. Our experiment provides the first empirical evidence supporting the real-life application of the celebrated Selfridge-Conway cake-cutting procedure. We hope that our findings guide its practical implementation, in the light of the very successful implementations of other fair division protocols in online platforms such as Spliddit.com.

Some interesting options for future experiments are: (a) Check other cake-cutting procedures, in particular, procedures that guarantee additional properties such as equitability, truthfulness or 
Pareto-efficiency. Is the added complexity of these procedures justified? Do they yield a better user experience? (b) Check division of more realistic resources. For example, instead of showing the subjects artificial one-dimensional "cakes", one can show them real two-dimensional maps of land-estates. Fair division of land is an important issue in many inheritance and dissolution cases; can cake-cutting procedures be used to solve such issues in practice?

\section{REFERENCES}

[1] Georgy Artemov, Yeon-Koo Che, and Yinghua He. 2017. Strategic mistakes: Implications for market design research. unpublished (2017).

[2] Haris Aziz and Simon Mackenzie. 2016. A discrete and bounded envy-free cake cutting protocol for 4 agents. In Proceedings of the Symposium on Theory of Computing (STOC).

[3] Anna Bassi. 2006. An experimental study of fair division. unpublished (2006).

[4] Xiaohui Bei, Ning Chen, Guangda Huzhang, Biaoshuai Tao, and Jiajun Wu. 2017. Cake cutting: envy and truth. In Proceedings of the International foint Conference on Artificial Intelligence (IFCAI).

[5] Xiaohui Bei, Guangda Huzhang, and Warut Suksompong. 2018. Truthful fair division without free disposal, In Proceedings of the International Joint Conference on Artificial Intelligence (IJCAI). arXiv preprint arXiv:1804.06923.

[6] Steven Brams and David Denoon. 1997. Fair division: A new approach to the Spratly Islands controversy. International Negotiation 2, 2 (1997), 303-329.

[7] Steven Brams, Michael Jones, and Christian Klamler. 2006. Better ways to cut a cake. Notices of the AMS 53, 11 (2006), 1314-1321.

[8] Steven Brams, Michael Jones, and Christian Klamler. 2008. Proportional pie-cutting. International fournal of Game Theory 36, 3-4 (2008), 353-367.

[9] Steven Brams, Michael Jones, and Christian Klamler. 2011. Divide-and-conquer: A proportional, minimal-envy cakecutting algorithm. SIAM Rev. 53, 2 (May 2011), 291-307.

[10] Steven Brams, Michael Jones, and Christian Klamler. 2013. N-person cake-cutting: There may be no perfect division. Amer. Math. Monthly 120, 1 (2013), 35-47.

[11] Steven Brams and Alan Taylor. 1996. Fair division: From cake-cutting to dispute resolution. Cambridge University Press.

[12] Steven Brams and Jeffrey Togman. 1996. Camp David: Was the agreement fair? Conflict Management and Peace Science 15, 1 (1996), 99-112.

[13] Simina Brânzei, Ioannis Caragiannis, David Kurokawa, and Ariel Procaccia. 2016. An algorithmic framework for strategic fair division. In Proceedings of the Conference on Artificial Intelligence (AAAI).

[14] Simina Brânzei and Peter Miltersen. 2013. Equilibrium analysis in cake cutting. In Proceedings of the Conference on Autonomous Agents and Multi-Agent Systems (AAMAS).

[15] Marco Castillo and Ahrash Dianat. 2016. Truncation strategies in two-sided matching markets: Theory and experiment. Games and Economic Behavior 98 (2016), 180-196.

[16] Ruggiero Cavallo. 2012. Fairness and welfare through redistribution when utility is transferable. In Proceedings of the Conference on Artificial Intelligence (AAAI).

[17] Yiling Chen, John Lai, David Parkes, and Ariel Procaccia. 2013. Truth, justice, and cake cutting. Games and Economic Behavior 77, 1 (2013), 284-297.

[18] Terry Daniel and James Parco. 2005. Fair, efficient and envy-free bargaining: An experimental test of the Brams-Taylor adjusted winner mechanism. Group Decision and Negotiation 14, 3 (May 2005), 241-264.

[19] Terry Daniel, Darryl Seale, and Amnon Rapoport. 1998. Strategic play and adaptive learning in the sealed-bid bargaining mechanism. Marketing Working Paper Series; MKTG 98.106 (1998).

[20] Payam Delgosha and Amin Gohari. 2012. Information theoretic cutting of a cake. In Proceedings of the Information Theory Workshop (ITW).

[21] John Dickerson, Jonathan Goldman, Jeremy Karp, Ariel Procaccia, and Tuomas Sandholm. 2014. The computational rise and fall of fairness.. In Proceedings of the Conference on Artificial Intelligence (AAAI).

[22] Lester Dubins and Edwin Spanier. 1961. How to cut a cake fairly. Amer. Math. Monthly 68, 1 (1961), 1-17.

[23] Nicolas Dupuis-Roy and Frédéric Gosselin. 2009. An empirical evaluation of fair-division algorithms. In Proceedings of the Annual Meeting of the Cognitive Science Society. 2681-2686.

[24] Nicolas Dupuis-Roy and Frédéric Gosselin. 2011. The Simpler, the Better: A New Challenge for Fair-Division Theory. In Proceedings of the Annual Meeting of the Cognitive Science Society. 3229-3234.

[25] Dirk Engelmann and Martin Strobel. 2004. Inequality aversion, efficiency, and maximin preferences in simple distribution experiments. American Economic Review 94, 4 (2004), 857-869.

[26] Shimon Even and Azaria Paz. 1984. A note on cake cutting. Discrete Applied Mathematics 7, 3 (1984), 285 - 296. 
[27] Ernst Fehr, Michael Naef, and Klaus Schmidt. 2006. Inequality aversion, efficiency, and maximin preferences in simple distribution experiments: Comment. American Economic Review 96, 5 (2006), 1912-1917.

[28] Merrill Flood. 1958. Some experimental games. Management Science 5, 1 (1958), 5-26.

[29] Duncan Foley. 1967. Resource allocation in the public sector. Yale Economic Essays 7 (1967), 73-76.

[30] Ya'akov Gal, Moshe Mash, Ariel Procaccia, and Yair Zick. 2016. Which is the fairest (rent division) of them all?. In Proceedings of the ACM Conference on Economics and Computation (EC).

[31] George Gamow and Marvin Stern. 1958. Puzzle-Math. Viking Press, New York.

[32] Jonathan Goldman and Ariel D Procaccia. 2015. Spliddit: Unleashing fair division algorithms. ACM SIGecom Exchanges 13, 2 (2015), 41-46.

[33] Werner Güth. 1995. On ultimatum bargaining experiments-A personal review. Fournal of Economic Behavior \& Organization 27, 3 (1995), 329-344.

[34] Avinatan Hassidim, Déborah Marciano, Assaf Romm, and Ran Shorrer. 2017. The mechanism is truthful, Why aren't you? American Economic Review 107, 5 (2017), 220-24.

[35] Avinatan Hassidim, Assaf Romm, and Ran I Shorrer. 2016. Strategic behavior in a strategy-proof environment. In Proceedings of the ACM Conference on Economics and Computation (EC).

[36] Dorothea K Herreiner and Clemens Puppe. 2007. Distributing indivisible goods fairly: Evidence from a questionnaire study. Analyse \& Kritik 29, 2 (2007), 235-258.

[37] Dorothea K Herreiner and Clemens Puppe. 2010. Inequality aversion and efficiency with ordinal and cardinal social preferences-An experimental study. Journal of Economic Behavior \& Organization 76, 2 (2010), 238-253.

[38] Dorothea K Herreiner and Clemens D Puppe. 2009. Envy freeness in experimental fair division problems. Theory and Decision 67, 1 (2009), 65-100.

[39] Rafael Hortala-Vallve and Aniol Llorente-Saguer. 2010. A simple mechanism for resolving conflict. Games and Economic Behavior 70, 2 (Nov. 2010), 375-391.

[40] Giuseppe Lopomo and Efe Ok. 2001. Bargaining, interdependence, and the rationality of fair division. RAND fournal of Economics 32, 2 (2001), 263-283.

[41] Tansa Massoud. 2000. Fair division, adjusted winner procedure (AW), and the Israeli-Palestinian conflict. Fournal of Conflict Resolution 44, 3 (June 2000), 333-358.

[42] Avishay Maya and Noam Nisan. 2012. Incentive compatible two player cake cutting. In Proceedings of the Workshop on Internet and Network Economics (WINE).

[43] Vijay Menon and Kate Larson. 2017. Deterministic, strategyproof, and fair cake cutting. In Proceedings of the foint Conference on Artificial Intelligence (IfCAI).

[44] Elchanan Mossel and Omer Tamuz. 2010. Truthful fair division. In Algorithmic Game Theory, Spyros Kontogiannis, Elias Koutsoupias, and Paul Spirakis (Eds.). Springer, 288-299.

[45] Hervé Moulin. 1995. Cooperative microeconomics: a game-theoretic introduction. Princeton University Press.

[46] Hervé Moulin. 2004. Fair division and collective welfare. MIT press.

[47] Antonio Nicolò and Yan Yu. 2008. Strategic divide and choose. Games and Economic Behavior 64, 1 (2008), 268 - 289.

[48] James Parco and Amnon Rapoport. 2004. Enhancing honesty in bargaining under incomplete information: An experimental study of the bonus procedure. Group Decision and Negotiation 13, 6 (2004), 539-562.

[49] Parag Pathak. 2018. What really matters in designing school choice mechanisms. Advances in Economics and Econometrics forthcoming (2018).

[50] John Pratt and Richard Zeckhauser. 1990. The fair and efficient division of the Winsor family silver. Management Science 36, 11 (Nov. 1990), 1293-1301.

[51] Ariel Procaccia. 2016. Cake cutting algorithms. In Handbook of Computational Social Choice, Felix Brandt, Vincent Conitzer, Ulle Endriss, Jerome Lang, and Ariel Procaccia (Eds.). Cambridge University Press, 311-330.

[52] Alex Rees-Jones. 2017. Suboptimal behavior in strategy-proof mechanisms: Evidence from the residency match. Games and Economic Behavior (2017).

[53] Jack Robertson and William Webb. 1998. Cake-cutting algorithms: Be fair if you can. AK Peters/CRC Press.

[54] Gerald Schneider and Ulrike Krämer. 2004. The limitations of fair division. Journal of Conflict Resolution 48, 4 (2004), 506-524.

[55] Hugo Steinhaus. 1948. The problem of fair division. Econometrica 16, 1 (1948), 101-104.

[56] Omer Tamuz, Shai Vardi, and Juba Ziani. 2018. Non-exploitable protocols for repeated cake cutting. In Proceedings of the Conference on Artificial Intelligence (AAAI).

[57] Stef Tijs and Rodica Branzei. 2004. Cases in cooperation and cutting the cake. SSRN preprint (Dec. 2004).

[58] Toby Walsh. 2011. Online cake cutting. Algorithmic Decision Theory 6992 (2011), 292-305.

[59] Güth Werner, Radosveta Ivanova-Stenzel, Manfred Königstein, and Martin Strobel. 2003. Learning to bid - an experimental study of bid function adjustments in auctions and fair division games. Economic fournal 113, 487 (2003), 477-494. 


\section{APPENDIX 1: ROBUSTNESS TESTS}

Envy. In the main text we classified a cake division as envious as long as the subject obtained fewer points than any other agent (i.e. preferred the piece of cake received by any other agent over hers). We found that envy-free procedures (2ACC, 2SCC, 3SC) produced significantly less envy than proportional ones. We also found that $4 \mathrm{EP}$ produced envy in most cases. Here, we changed the definition of envy as a robustness test. We say that the subject envies an agent only if the number of points obtained by the subject plus some number $x$ is smaller than the number of points received by the agent, where $x$ is either 5 or 10 . We refer to these cases as envy $(+5)$ or envy $(+10)$. For example, if the subject obtained 50 points but another subject obtained 53, we would say that the agent is envious in the main text but not in this robustness test.

Table 4 presents an overview of our findings with this updated definition of envy $(+5)$. We do not present the results for envy $(+10)$ as they are almost identical.

Table 4. Percentage of cases where envy $(+5)$ is generated, by round.

\begin{tabular}{llllll|l|ll|l|l}
\hline \hline $\begin{array}{c}\text { Round } \\
\text { Proc }\end{array}$ & 1 & 2 & 3 & 4 & 5 & $\begin{array}{l}\text { No knowl. } \\
\text { average }\end{array}$ & 6 & 7 & $\begin{array}{l}\text { Knowl. } \\
\text { average }\end{array}$ & $\begin{array}{l}\text { Total } \\
\text { average }\end{array}$ \\
\hline 2ACC & 10 & 8 & 5 & 5 & 6 & 7 & 5 & 3 & 4 & 6 \\
2SCC & 16 & 24 & 23 & 20 & 13 & 19 & 16 & 14 & 15 & 18 \\
3DS & 56 & 67 & 62 & 60 & 57 & 60 & 72 & 68 & 70 & 63 \\
3LD & 55 & 51 & 49 & 52 & 42 & 50 & 44 & 40 & 42 & 48 \\
3SC & 27 & 23 & 24 & 26 & 30 & 26 & 23 & 23 & 23 & 25 \\
4DS & 61 & 85 & 83 & 77 & 76 & 76 & 77 & 72 & 75 & 76 \\
4LD & 61 & 64 & 52 & 53 & 49 & 56 & 53 & 44 & 49 & 54 \\
4EP & 93 & 86 & 90 & 87 & 86 & 88 & 88 & 83 & 86 & 88 \\
\hline \hline
\end{tabular}

We observe that all proportional procedures still generate envy in half or more of the cases. We observe that 3SC generates substantially less envy than 3LD and 3DS. Similarly, we observe that $4 \mathrm{EP}$ is by far the procedure that generates more envy. Thus we conclude that our findings regarding envy are robust to the exact definition of envy.

Manipulations. In the main text we defined manipulations as deviations within 5 score points. Here, as robustness tests, we define manipulations as deviations within 10 and 15 points. We first present the percentage of truthful behavior, and of successful and unsuccesful manipulations in Figures $\mathbf{5 a}$ and $\mathbf{5 b}$, respectively. The conclusions presented in the main text still apply: we observe a large number of scores obtained by both the successful and unsuccessful manipulation of preferences.

We check in both cases whether the number of non-truthful reports increases as agents learn more about their opponents' preferences. We found that this was the case in the main text. We conduct a logit regression for repeated measures of the probability of playing truthful strategies (under both definitions) on the round number. For the case of manipulations within 10 points, we obtain a coefficient of -.11 that is statistically significant (p-value $<0.001$ ). For the case of manipulations within 15 points, we obtain similar results (a coefficient of -.1 with a p-value < 0.001). Thus, we conclude that our finding that truthful play decreases over time is robust to the exact definition of truthful behavior.

Manipulations: Looking at Cake Cuts instead of Utilities. In the main text we identified manipulations by looking at changes in the utilities of the subjects. We found evidence of strategic behavior 


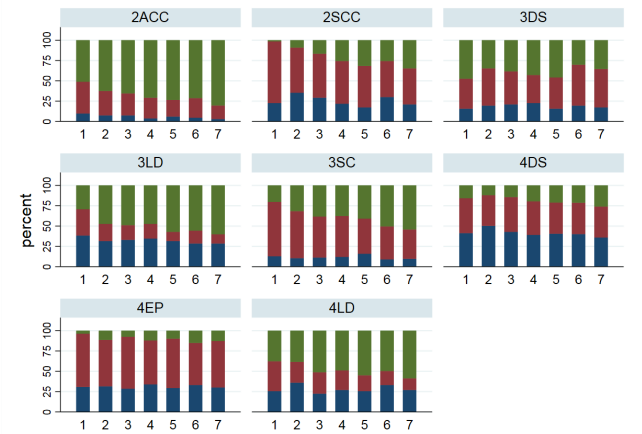

(a) Manipulations \pm 10 points

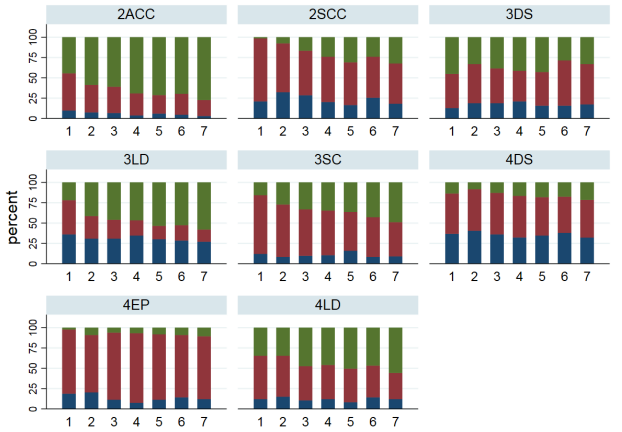

(b) Manipulations \pm 15 points

Fig. 5. Percentage of truth-telling (red) and successful (green) and unsuccessful (blue) manipulations, by procedure. Manipulations are defined as deviations \pm 10 (a) and 15 (b) points from truth-telling.

in all procedures. Here we take the alternative route of looking at the actual cake cuts that subjects made. We have this data based on the exact pixel that subjects choose to cut the cake. We give a \pm 5 pixels deviation tolerance. These results are presented in Figure 6, in which we describe the percentage of truthful cake cuts (since in some procedures the agents need to make more than one cut, we examine only the truthfulness of the first cut).
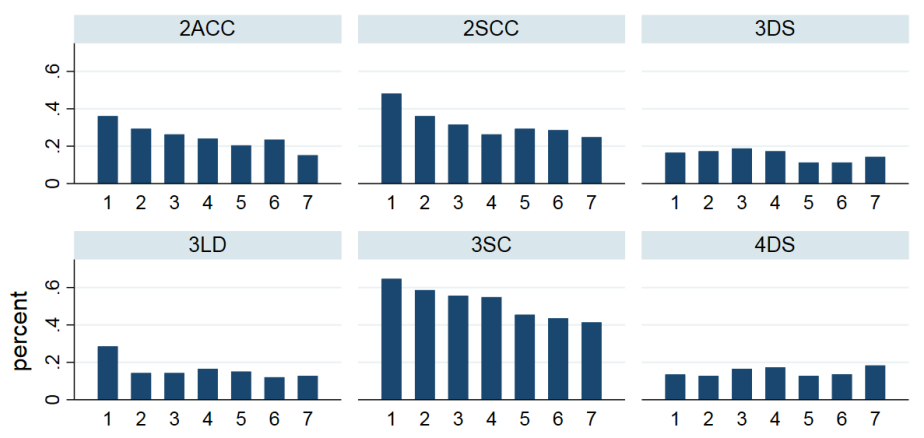

$3 \mathrm{SC}$
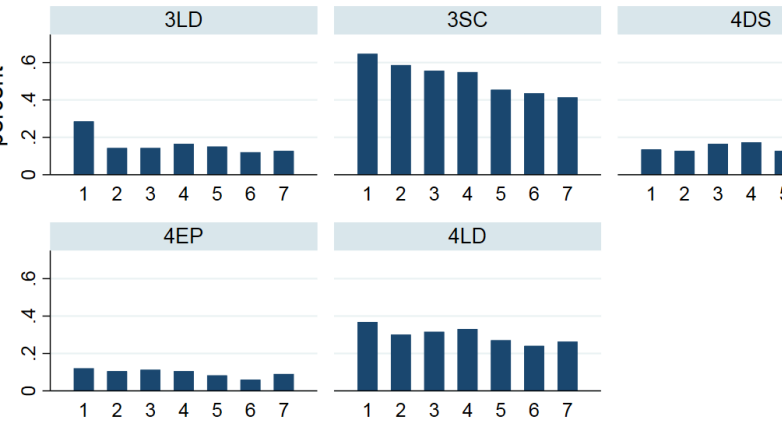

Fig. 6. Percentage of truthful cake cuts, by procedure over rounds.

We observe that our main finding is robust: we still observe that agents make strategic cake cuts most of the times. However, we observe one difference with respect to the analysis of utilities. In the case of utilities, we observe truthful behavior in about $50 \%$ of the cases, whereas if we look at cake cuts, we observe that truth-telling only occurs in about $10 \%$ of cases. Looking at utilities does not allow us to identify a large number of manipulations which are payoff irrelevant in the 
case of 4EP. For the other procedures, examining manipulation via utilities or cake cuts leads to similar conclusions. We also perform a logit regression for repeated measures of the probability of playing truthful strategies (using cake cuts) on the round number. The results are very similar as in the previous cases: the corresponding coefficient is -0.11 and is statistically significant ( $\mathrm{p}$-value $<0.001)$. 


\section{APPENDIX 2: PREFERENCE PROFILES USED}

All the preference profiles are generated using piecewise uniform valuations. The cake is divided in 600 pixels of equal length with each desired pixel giving the agent 1 point. Agents desire 120 pixels which give the corresponding 120 points described in the main text. We present the preferences using the tables below; a one in the table indicates that the agent desires the interval in question. The intervals that are not mentioned are not desired by any agent. These can also be downloaded from here, which may be easier to read.

Table 5. Preferences used in 2ACC.

\begin{tabular}{|l|l|l|l|l|l|l|}
\hline \hline 2ACC & $61-120$ & $121-130$ & $171-190$ & $291-310$ & $411-430$ & $451-540$ \\
\hline Subject & 1 & 0 & 1 & 1 & 1 & 0 \\
Robot 1 & 0 & 1 & 0 & 0 & 1 & 1 \\
\hline \hline
\end{tabular}

Table 6. Preferences used in 2SCC.

\begin{tabular}{|l|l|l|l|l|l|l|l|l|l|l|}
\hline \hline 2SCC & $141-170$ & $191-220$ & $231-240$ & $241-260$ & $271-300$ & $311-320$ & $321-330$ & $361-390$ & $471-490$ & $511-540$ \\
\hline Subject & 0 & 0 & 1 & 1 & 1 & 1 & 0 & 0 & 1 & 1 \\
Robot 1 & 1 & 1 & 1 & 0 & 0 & 1 & 1 & 1 & 0 & 0 \\
\hline \hline
\end{tabular}

Table 7. Preferences used in 3DS.

\begin{tabular}{|l|l|l|l|l|l|l|l|l|l|l|}
\hline \hline 3DS & $71-110$ & $121-130$ & $131-150$ & $151-160$ & $171-180$ & $191-200$ & $271-310$ & $311-380$ & $411-430$ & $451-540$ \\
\hline Subject & 1 & 1 & 1 & 1 & 0 & 0 & 1 & 0 & 0 & 0 \\
Robot 1 & 0 & 1 & 0 & 0 & 0 & 0 & 0 & 0 & 1 & 1 \\
Robot 2 & 0 & 1 & 1 & 0 & 1 & 1 & 0 & 1 & 0 & 0 \\
\hline \hline
\end{tabular}

Table 8. Preferences used in 4DS.

\begin{tabular}{|l|l|l|l|l|l|l|l|l|l|l|l|l|l|l|l|l|l|l|l|}
\hline \hline 4DS & $61-80$ & $81-90$ & $91-120$ & $141-150$ & $151-170$ & $171-180$ & $181-210$ & $211-240$ & $241-270$ & $271-300$ & $301-330$ & $331-360$ & $371-390$ & $391-420$ & $421-450$ & $451-480$ & $491-510$ & $511-540$ \\
\hline Subject & 1 & 0 & 0 & 1 & 1 & 0 & 0 & 0 & 1 & 0 & 0 & 0 & 1 & 0 & 0 & 0 & 1 & 0 \\
Robot 1 & 1 & 1 & 0 & 0 & 0 & 0 & 1 & 0 & 0 & 0 & 1 & 0 & 0 & 0 & 1 & 0 & 0 & 0 \\
Robot 2 & 0 & 0 & 1 & 0 & 0 & 0 & 0 & 1 & 0 & 0 & 0 & 1 & 0 & 0 & 0 & 1 & 0 \\
Robot 3 & 0 & 0 & 0 & 0 & 1 & 1 & 0 & 0 & 0 & 1 & 0 & 0 & 0 & 1 & 0 \\
\hline \hline
\end{tabular}

Table 9. Preferences used in 3LD.

\begin{tabular}{|l|l|l|l|l|l|l|l|l|l|l|l|l|l|}
\hline \hline 3LD & $71-90$ & $91-110$ & $121-190$ & $221-230$ & $231-260$ & $281-300$ & $301-320$ & $341-350$ & $351-370$ & $371-400$ & $401-410$ & $431-440$ & $451-460$ \\
\hline Subject & 0 & 1 & 0 & 1 & 1 & 1 & 0 & 1 & 1 & 0 & 1 & 0 & 0 \\
Robot 1 & 1 & 1 & 1 & 1 & 0 & 0 & 0 & 0 & 0 & 0 & 0 & 0 & 0 \\
Robot 2 & 0 & 0 & 0 & 0 & 0 & 1 & 1 & 0 & 1 & 1 & 1 & 1 & 1 \\
\hline \hline
\end{tabular}


Table 10. Preferences used in 4LD.

\begin{tabular}{|l|l|l|l|l|l|l|l|l|l|l|l|l|l|l|}
\hline \hline 4LD & $61-90$ & $91-110$ & $111-160$ & $181-230$ & $231-250$ & $251-270$ & $271-280$ & $281-290$ & $311-340$ & $341-350$ & $351-370$ & $371-380$ & $381-410$ & $421-520$ \\
\hline Subject & 1 & 0 & 0 & 1 & 1 & 0 & 1 & 1 & 0 & 0 & 0 & 0 & 0 & 0 \\
Robot 1 & 0 & 0 & 1 & 0 & 0 & 1 & 1 & 0 & 0 & 1 & 1 & 1 & 0 & 0 \\
Robot 2 & 0 & 0 & 0 & 0 & 1 & 1 & 0 & 0 & 1 & 1 & 0 & 1 & 1 & 0 \\
Robot 3 & 0 & 1 & 0 & 0 & 0 & 0 & 0 & 0 & 0 & 0 & 0 & 0 & 0 & 1 \\
\hline \hline
\end{tabular}

Table 11. Preferences used in 4EP.

\begin{tabular}{|l|l|l|l|l|l|l|l|l|l|l|l|l|l|l|l|l|l|}
\hline \hline 4EP & $11-110$ & $111-120$ & $121-140$ & $161-170$ & $171-190$ & $191-210$ & $211-220$ & $221-240$ & $241-270$ & $281-300$ & $301-320$ & $331-340$ & $341-350$ & $351-360$ & $361-370$ & $411-430$ & $471-510$ \\
\hline Subject & 1 & 1 & 0 & 0 & 1 & 1 & 1 & 1 & 0 & 0 & 1 & 0 & 0 & 0 & 0 & 0 & 0 \\
Robot 1 & 0 & 1 & 1 & 0 & 1 & 1 & 0 & 0 & 0 & 1 & 1 & 0 & 0 & 1 & 0 & 0 & 0 \\
Robot 2 2 & 0 & 0 & 0 & 0 & 0 & 1 & 1 & 0 & 0 & 1 & 1 & 0 & 1 & 1 & 1 & 1 \\
Robot 3 & 0 & 0 & 0 & 1 & 1 & 0 & 0 & 0 & 1 & 0 & 0 & 1 & 1 & 0 & 0 & 0 \\
\hline \hline
\end{tabular}

Table 12. Preferences used in 3SC.

\begin{tabular}{|l|l|l|l|l|l|l|l|l|l|l|l|l|l|l|l|l|l|l|}
\hline \hline $3 S C$ & $71-80$ & $81-90$ & $91-100$ & $101-110$ & $141-150$ & $151-170$ & $171-190$ & $211-230$ & $271-280$ & $281-290$ & $291-300$ & $301-320$ & $321-330$ & $331-340$ & $381-400$ & $451-470$ & $471-490$ \\
\hline Subject & 0 & 0 & 0 & 0 & 0 & 1 & 1 & 1 & 0 & 0 & 0 & 0 & 0 & 0 & 1 & 1 & 1 \\
Robot 1 & 0 & 1 & 0 & 1 & 1 & 1 & 0 & 0 & 1 & 1 & 0 & 1 & 0 & 1 & 0 & 0 & 1 \\
Robot 2 & 1 & 1 & 1 & 1 & 0 & 0 & 0 & 0 & 0 & 1 & 1 & 1 & 1 & 1 & 0 & 0 \\
\hline
\end{tabular}




\section{APPENDIX 3: EXPERIMENT INSTRUCTIONS}

Upon their arrival to the lab, the cake-cutting procedures are explained to the subjects using the slides available here. We do not include them here for the sake of brevity. The presentation comprises 31 slides to make the procedures as clear as possible. We describe the text that the subjects observe in the graphical interface. These are as follows:

Welcome to the game. When you are ready to start click the start button.

First tab: I Cut You choose, against 1 opponent. Description: You will cut the cake into two parts. Your opponent will choose the one he prefers. You will receive the other one. Suggestion: If you cut the cake in two pieces worth 60 points, you guarantee that you will receive 60 points. Dividing the cake differently may give you more points, but may also give you less.

Second tab: Cut Middle, against 1 opponent. Description: You will cut the cake into two parts. Your opponent also cuts the cake into two. We cut the cake in the middle of those cuts and you get the part that includes your cut. Suggestion: If you cut the cake in two pieces worth 60 points, you guarantee that you will receive at least 60 points. Dividing the cake differently may give you more points, but may also give you less.

Third tab: Leftmost Leaves, against 2 opponents. Description: All players make one cut to the cake. The one who cuts the leftmost piece gets that part and leaves. The procedure is repeated until no agent is left. You may need to cut the cake twice in the same round if you don't choose the leftmost piece right away. Suggestion: If you cut the cake at 40 in each stage, you guarantee at least 40 points. Dividing the cake differently may give you more points, but may also give you less.

Fourth tab: Leftmost Leaves, against 3 opponents. Description: All players make one cut to the cake. The one who cuts the leftmost piece gets that part and leaves. The procedure is repeated until no agent is left. You may need to cut the cake twice in the same round if you don't choose the leftmost piece right away. Suggestion: If you cut the cake at 30 in each stage, you guarantee at least 30 points. Dividing the cake differently may give you more points, but may also give you less.

Fifth tab: Last Challenger, against 2 opponents. Description: You make a cut to the cake. This cut can be challenged by other players. If it is not challenged, you get the left piece of the cake and leave. If it is challenged, the player who challenges gets the left piece and leaves, and we restart the procedure with the leftover cake. You may need to cut the cake twice in the same round if your initial cut is challenged. Suggestion: If you cut the cake at 40 in each stage, you guarantee at least 40 points. Dividing the cake differently may give you more points, but may also give you less.

Sixth tab: Last Challenger, against 3 opponents. Description: You make a cut to the cake. This cut can be challenged by other players. If it is not challenged, you get the left piece of the cake and leave. If it is challenged, the player who challenges gets the left piece and leaves, and we restart the procedure with the leftover cake. You may need to cut the cake twice in the same round if your initial cut is challenged. Suggestion: If you cut the cake at 30 in each stage, you guarantee at least 30 points. Dividing the cake differently may give you more points, but may also give you less.

Seventh tab: Super Fast, against 3 opponents. Description: All players split the cake into two. The two who choose the leftmost cuts divide the first half, the other two the second half. Each half is divided using leftmost leaves. You will have to cut the cake twice. Suggestion: If you first cut the 
cake at 60 points and then at 30 , you guarantee at least 30 points. Dividing the cake differently may give you more points, but may also give you less.

Eight tab: Super Fair, against 2 opponents. Description: In this procedure you have two knives. You should cut the cake into three pieces. Then a complex procedure occurs, which you can read in your information sheet. Suggestion: If you cut the cake into three pieces worth 40 points each, you guarantee 40 points. Dividing the cake differently may give you more points, but may also give you less.

Additional Explanation for 3SC. You will cut the cake into three pieces using two knives. We suggest you to cut the cake into three pieces worth 40 points each so to guarantee yourself 40 points. Dividing the cake differently may give you more points, but may also give you less.

After you cut the cake, opponent 1 will trim her most valued piece so to make her two most preferred pieces of equal value. The part she cuts from her most valued piece of cake will be put apart and divided later (the trimmings). Then opponent 2 will take the part he prefers. If opponent 2 does not take the part that opponent 1 trims, then opponent 1 will receive that part and you will receive the leftover. Otherwise, in case opponent 2 picks the trimmed part, opponent 1 chooses one of the two remaining pieces and then you choose last.

Once the main pieces of the cake have been divided, we will divide the trimmings. One of the two opponents (the one who did not choose the trimmed part) will cut the trimmings into three pieces. Then the other opponent will choose one of them. From the two leftovers, you will be given the one which is best for you, and the last one will be given to the remaining opponent.

Subjects also receive an official information sheet with the following information: Strategic Behavior in Fair Division Problems

Invitation to our study. We would like to invite you to participate in this research project. You should only participate if you want to; choosing not to take part will not disadvantage you in any way. Before you decide whether you want to take part, it is important for you to read the following information carefully and discuss it with others if you wish. Ask us if there is anything that is not clear or you would like more information.

Background on the project. We are conducting an exploration of how people make economic decisions, in particular on how they decide to divide and share resources with others. We are testing how different resource allocation methods affect the economic decisions people make.

Experiment. You will be asked to divide resources with 2,3, or 4 other agents. The way in which you decide to divide the resources will affect how much money you will receive by the end of the experiment. The experiment will last for around one hour. You won't be required to participate again in the experiment. You will be paid in private at the end of the experiment. You will receive at least $\$ 5$ for showing up, but you may earn more money based on your decisions throughout this session.

Are there any risks associated with this experiment? There are no risks associated with this experiment. Shall you experience any discomfort please contact any member of the staff.

Informed consent. Should you agree to take part in this experiment, you will be asked to sign a consent form before the experiment commences.

Withdrawal. Your participation is voluntary and you will be free to withdraw from the project at any time without giving any reason and without penalty. If you wish to withdraw, you simply need to notify the principal investigator (see contact details below). If any data have already been 
collected, upon withdrawal, your data will be destroyed, unless you inform the principal investigator that you are happy for us to use such data for the scientific purposes of the project.

Data gathered. We will record the economic decisions you make during the experiment, namely how you decide to share resources with other participants. Signed consent forms will be kept separately from individual experimental data and locked in a drawer until the end of the project.

Findings. After the end of the project, we will publish the findings of our research. We will be happy to provide you with a lay summary of the main findings and with copies of the articles published if you express an interest.

Concerns and complaints. If you have any concerns about any aspect of the study or you have a complaint, in the first instance please contact the principal investigator of the project (see contact details below). If are still concerned or you think your complaint has not been addressed to your satisfaction, please contact the Director of Research in the principal investigator's department (see below). If you are still not satisfied, please contact the University's Research Governance and Planning Manager.

Funding. Corresponding details.

Ethical approval. This project has been reviewed on behalf of the University Ethics Committee and had been given approval.

Principal investigator. Corresponding details.

Finally, we include the questions in the fairness survey that subjects complete after they complete the cutting of all the cakes.

Experiment feedback. Please answer (with as many details as possible) the following questions.

How fair was "Cut and choose"? Very unfair, Unfair, Fair, Very fair.

Feedback: textbox.

How fair was "middle cut"? Very unfair, Unfair, Fair, Very fair.

Feedback: textbox.

How fair was "last challenger"? Very unfair, Unfair, Fair, Very fair.

Feedback: textbox.

How fair was "lefmost leaves"? Very unfair, Unfair, Fair, Very fair.

Feedback: textbox.

In your opinion, was "super fair" a fairer procedure than all the others? Yes, No.

Feedback: textbox.

In your opinion, was "super fast" an easier procedure to use than all the others? Yes, No.

Feedback: textbox.

Would you have preferred to bargain over the cake directly with the other players instead of dividing it with these methods? Yes, No, Doesn't matter.

Please give us your comments on which procedures produced fairer allocations and were easier to use.

Feedback: textbox. 


\section{APPENDIX 4: OMITTED PROOFS}

We present the proofs omitted in the main text.

LEMma (3). The procedures $2 A C C, 2 S C C, n D S, n L D, n E P, 3 S C$ are $\frac{n-1}{n}$ strategy-proof and this is tight.

Proof of Lemma 3. To prove that $\epsilon=\frac{n-1}{n}$ is tight, we provide instances such that an agent would get utility exactly $1 / n$ by truthfully reporting her valuation function, while she could get utility 1 by strategizing.

We start with the case of 3SC. Consider a cake $[0,1]$ and the following valuations of the agents: $v_{1}(0,1 / 3)=v_{2}(1 / 3,2 / 3)=v_{3}(2 / 3,1)=1$; agents have valuation 0 for any other part. Assume everyone behaves truthfully, and in the first step agent 1 divides the cake in the following parts of equal value to him: $[0,1 / 9),[1 / 9,2 / 9)$, and $[2 / 9,1]$. Agent 2 has positive valuation only for the last part, so in the next step, she will trim it so that the trimmed part has value 0 to him; let's assume that the trimmed part is negligible and has value 0 to everyone. Agent 3 now has to select one of the parts for which she is indifferent (she only values the trimmings), so let him select the part $[1 / 9,2 / 9)$, let agent 2 take the trimmed part, and let agent 1 get the $[0,1 / 9)$ part. Agent 3 will now divide the trimmings so that each part is worth $1 / 3$ to him, namely $(2 / 9,7 / 9),[7 / 9,8 / 9)$, and $[8 / 9,1]$. Agent 2 will rationally select the first one of these parts, leaving agent 1 with an overall utility of $1 / 3$, after the end of the process.

Now imagine that agent 1 behaves strategically in the first step and divides the cake into the parts $[0,1 / 3),[1 / 3,2 / 3)$, and $[2 / 3,1]$. Agent 2 will trim the second part so that the trimmed part is negligible, i.e. it is worth 0 to everyone. Agent 3 will rationally get her desired part, i.e. [2/3, 1], agent 2 will get the trimmed part, and agent 1 will get her desired part, i.e. $[0,1 / 3)$. Now, let agent 3 divide the trimmings as follows (recall that she is indifferent because the trimmings are worth 0 to him): one part is $(1 / 3,2 / 3)$, and the other parts are negligible. Agent 2 will now rationally select this first part in the next step and, overall, each agent gets utility 1 in this case where only agent 1 strategizes.

Regarding 2SCC, consider a cake $[0,1]$ and the following valuations of the agents, where $\epsilon>0$ is negligibly small: $v_{1}(0,1 / 4)=v_{1}(1 / 2-2 \epsilon, 1 / 2-\epsilon)=\frac{1}{2}$, and $v_{2}(1 / 2,1 / 2+\epsilon)=v_{2}(1 / 2+\epsilon, 1 / 2+2 \epsilon)=\frac{1}{2}$; agents have valuation 0 for any other part. Assume everyone behaves truthfully, and agents 1 and 2 cut the cake at points $1 / 4$ and $1 / 2+\epsilon$, respectively, to divide it to two parts of equal value to them. After the end of the procedure agent 1 will receive utility $1 / 2$. Now imagine that agent 1 behaves strategically and cuts the cake at $1 / 2-\epsilon$. In the resulting allocation each of the agents will receive utility 1.

The other cases are simpler and use instances with valuation functions of the form $v_{i}\left(\frac{i-1}{n}, \frac{i}{n}\right)=1$ for $i=1, \ldots, n$, and 0 otherwise, similar to 3SC. The analysis is straightforward (similar, yet much simpler than the one for 3SC), hence we omit it.

Lemma (4). Envy can be generated in 3SC with just one agent misrepresenting her preferences. This agent achieves a higher payoff at the cost of being envious.

Proof. We present an instance and a corresponding strategy for agent 1 who is assumed to be strategic and tries to maximize her utility when competing with two truthful agents. We show that agent 1 will end up envious of another agent, although he will achieve higher utility than what he would get by behaving truthfully. We focus on the action of agent 1 at the beginning of the process, when he is asked to split the cake in three pieces. We consider this to be the strategy of agent 1 ; w.l.o.g. we ignore subsequent actions in the analysis as the only other choice that agent 1 makes is to select a part of the trimmings close to the end of the process, and it is clear that her incentives at that point are aligned with behaving truthfully and getting the part that is most valuable to her. 
Consider a cake $[0,1]$, which comprises 6 parts. The preferences of the agents are described by the valuations in Table 13; agents are assumed to have uniform valuations within each of these parts. $\epsilon$ denotes a very small positive constant.

Table 13. Agents' preferences such that under 3SC agent 1's optimal strategy makes her envious.

\begin{tabular}{|l|c|c|c|c|c|c|}
\hline \hline & 1 & 2 & 3 & 4 & 5 & 6 \\
\hline Agent 1 & 0 & $1 / 3$ & $\epsilon$ & $1 / 3-\epsilon$ & $\epsilon$ & $1 / 3-\epsilon$ \\
Agents 2 and 3 & $1 / 6$ & $1 / 6$ & $1 / 3$ & 0 & $1 / 3$ & 0 \\
\hline \hline
\end{tabular}

Truthful behavior for agent 1 would imply that he divides the cake at three equally valued pieces, i.e., the first piece contains parts 1 and 2, the second piece contains parts 3 and 4 , and the third piece contains parts 5 , and 6 . This split would result in utility $1 / 3$ for agent 1 as there would essentially be no trimming and each of the other agents would obtain one of these parts. We claim that the split in three pieces comprising parts $1-4,5$, and 6 , respectively, is an optimal strategy for agent 1 when others are truthful, under which he ends up getting utility strictly higher than $1 / 3$ but is envious of one of the other two agents.

We first show that the split in pieces 1-4, 5, and 6, is a better response for agent 1 than behaving truthfully, yet makes him envious of another agent. Indeed, under this split, agent 2 will trim the first piece so that the trimmings comprise parts 1 and 2. Agents 2 and 3 will first choose pieces 3-4 (the trimmed piece), and 5, respectively, leaving part 6 for agent 1 . The trimmings $T$ will be split in three equal parts, for which agent 1 will have value $0,1 / 9$, and $2 / 9$, respectively. Whoever got the trimmed piece, 3-4, will first select the third part of the trimmings, leaving the second one for agent 1. Overall, agent 1's allocation has value $\frac{1}{3}-\epsilon+\frac{1}{9}=\frac{4}{9}-\epsilon>\frac{1}{3}$, yet he is envious of the agent who got the trimmed part, as his allocated pieces have total value $\frac{1}{3}+\frac{2}{9}=\frac{5}{9}$ to agent 1 .

It remains to show that the split in pieces 1-4, 5, and 6 is an optimal strategy (the one that results in the highest utility) for agent 1 . We can prove this under certain assumptions on the behavior of the agents that guarantee consistency. The proof involves a case-analysis on all possible strategies of agent 1 . Recall that the cake $[0,1]$ comprises 6 parts. Agents are assumed to have uniform valuations within each of these parts. $\epsilon$ denotes a very small positive constant.

We first need to make some assumptions regarding the behavior of the agents (these assumptions also help so that the tie-breaking between equivalent truthful actions of agents 2 and 3 is consistent). We first assume that agents cannot cut in the interior of parts 1, 2, 3 and 5 (the instance could be defined so that these parts have a negligibly small width and the space of allowed cuts is discrete); for the smooth execution of the protocol we allow such cuts if and only if it is absolutely necessary in order to achieve an exact trimmed piece or an even distribution of trimmings. For consistency when trimming, we assume that the trimmings will be on the left part of the trimmed piece, unless a cut in the interior of the parts mentioned above can be avoided otherwise. Finally, among equivalent actions agents 2 and 3 will choose the one that harms agent 1 the most.

To see why the split in pieces $1-4,5$, and 6 is an equilibrium strategy for agent 1 we show that $4 / 9-\epsilon$ is the maximum utility she can get, by examining all other possible cuts she could make at the beginning of the procedure. Let $c_{1}$ and $c_{2}$ denote the cuts of agent $1, T$ denote the trimmings, 
and $t_{1}$ and $t_{2}$ denote the cuts that divide the trimmings. We denote by $c_{i} \in P$, for $i=1,2$ the fact that the $i$-th cut is in part $\mathrm{P}$ (or on its boundary), and we denote by $r_{j}, l_{j}$ the right and left boundary, respectively, of part $j$, for $j=1, \ldots, 6$.

- $c_{1}, c_{2} \in 6$. Under our tie-breaking assumptions, $T=\left[0, c_{1}\right)$ and agent 1 will get the least valuable piece among $\left[c_{1}, c_{2}\right)$ and $\left[c_{2}, 1\right]$ before the splitting of the trimmings. $T$ will be split by $t_{1}=l_{3}$ and $t_{2}=1 / 3+\frac{c_{1}-1 / 3}{2}$, and agent 1 will also get her second most valuable part of $\mathrm{T}$. In total, agent 1 will get at most $\frac{1-c_{1}}{2}+\frac{c_{1}-1 / 3}{2} \leq 1 / 3$.

- $c_{1} \in 4, c_{2} \in 6$. $T$ comprises parts $1-2$ and agent 1 will get part $\left[c_{2}, 1\right]$ before the splitting of the trimmings. $T$ will be split in three equal parts, for which agent 1 will have value $0,1 / 9$, and 2/9, respectively. Agent 1 will get her second most valuable part of T, and overall her allocation has value at most $\frac{1}{3}-\epsilon+\frac{1}{9}=\frac{4}{9}-\epsilon$.

- $c_{1}, c_{2} \in 4$. $T$ comprises parts 1-2 and agent 1 will get piece $\left[c_{1}, c_{2}\right)$ before the splitting of the trimmings. Similarly to the previous case, agent 1 will gain value $1 / 9$ from the trimmings which gives him at most $\frac{4}{9}-\epsilon$ in total.

- $c_{1}=l_{3}, c_{2} \in 6 . T=\left(c_{1}, r_{3}\right)$ and agent 1 gets $\left[c_{2}, 1\right]$ before the splitting of the trimmings. Agent 1 gets value less than $1 / 3$ in total.

- $c_{1}=l_{3}, c_{2} \in 4$. No trimming is required. Agent 1 will get value at most $1 / 3$.

- $c_{1}=l_{2}, c_{2} \in 6 . T=\left[l_{3}, c_{2}\right)$ and agent 1 gets $\left[c_{2}, 1\right]$ before the splitting of the trimmings, worth less than $1 / 3$ to her. One part of the trimmings will contain part 4 , and that part will be chosen first by the agent among 2 and 3 who got the trimmed part. Overall, agent 1 gets value less than $1 / 3$ in total.

- $c_{1}=l_{2}, c_{2} \in 4 . T=\left(c_{1}, r_{2}\right)$ and agent 1 gets $\left[0, c_{1}\right]$ before the splitting of the trimmings, worth 0 to her. Agent 1 gets value less than $1 / 3$ in total.

- $c_{1}=l_{2}, c_{2}=r_{2} . T=\left(c_{2}, z\right)$, where $z$ is in the middle of part 5 and agent 1 gets $\left[0, c_{1}\right]$ before the splitting of the trimmings, worth 0 to her. $t_{2}$ is in the middle of part 3 and $t_{3}=r_{3}$, and agent 1 will not get the part of the trimmings containing part 4 . Hence, agent 1 gets value less than $1 / 3$ in total.

- $c_{1}=l_{1}, c_{2} \in 6 . T=\left(c_{1}, c_{2}\right)$ and agent 1 gets $\left[0, c_{1}\right)$ before the splitting of the trimmings, worth 0 to her. $t_{1}=l_{3}$ and $t_{2}=r_{4}$ which means that agent 1 cannot get more than $1 / 3$ in total.

- $c_{1}=l_{1}, c_{2} \in 4 . T=\left(c_{1}, r_{2}\right)$ and agent 1 gets $\left[0, c_{1}\right)$ before the splitting of the trimmings, worth 0 to her. Agent 1 cannot get more than $1 / 3$ in total.

- $c_{1}=l_{1}, c_{2}=r_{2} . T=\left[c_{2}, r_{3}\right]$ and agent 1 gets $\left[0, c_{1}\right)$ before the splitting of the trimmings, worth 0 to her. Agent 1 cannot get more than $1 / 3$ in total.

- $c_{1}=l_{1}, c_{2}=r_{1} . T=\left[l_{3}, 1\right]$ and agent 1 gets $\left[0, c_{1}\right)$ before the splitting of the trimmings, worth 0 to her. $t_{1} \in 3$ and $t_{2} \in 5$ imply that agent 1 cannot get more than $1 / 3$ in total.

The proof is now complete. 


\section{APPENDIX 4: OMITTED FIGURES AND TABLES}

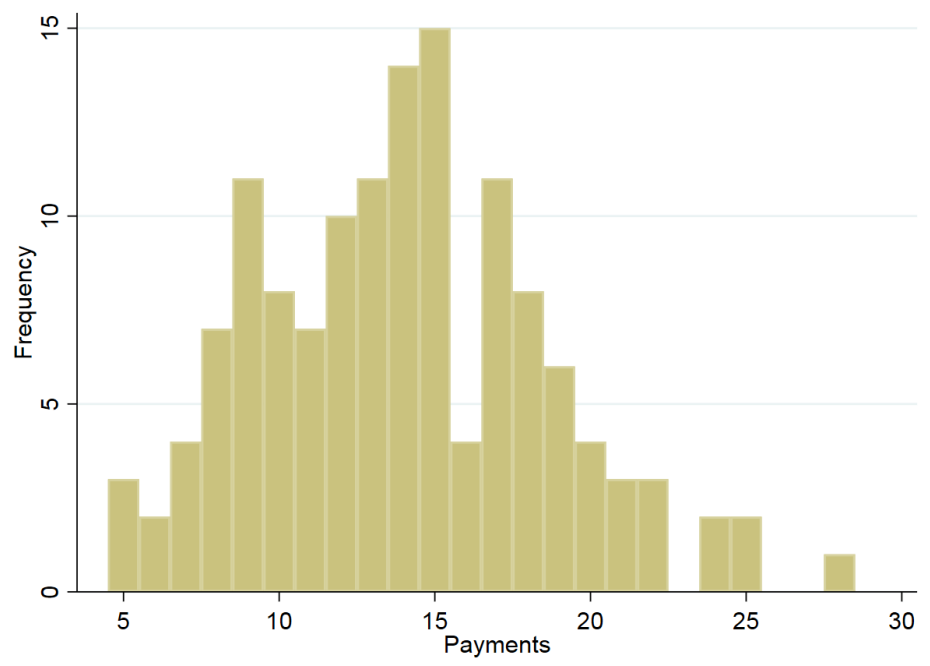

Fig. 7. Distribution of payments.

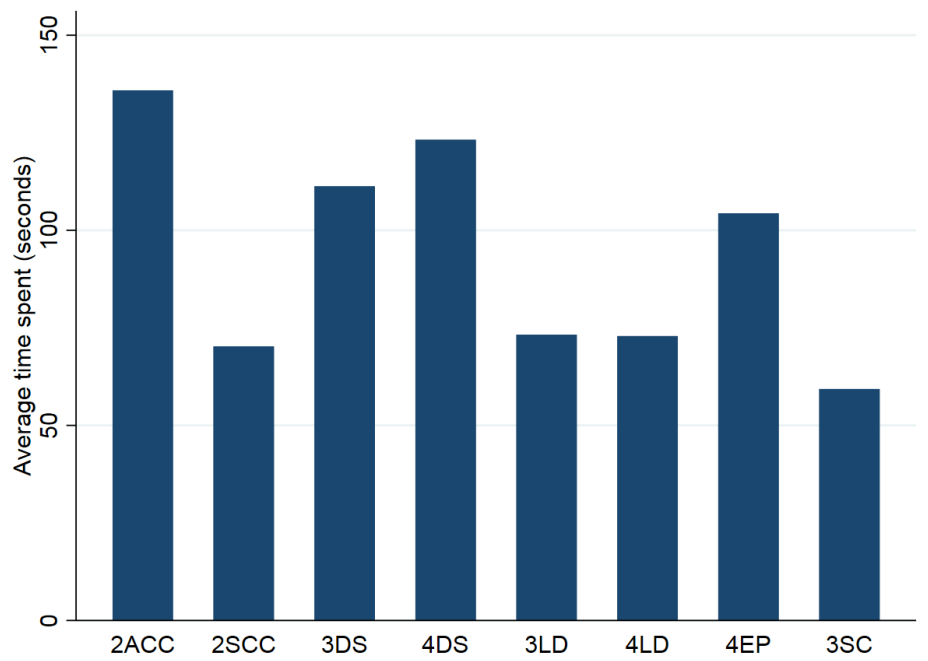

Fig. 8. Average time spent in each procedure. In seconds, procedures are displayed in order in which they are faced. 


\begin{tabular}{lcccccccc}
\hline \hline & 2ACC & 2SCC & 3DS & 3LD & 3SC & 4DS & 4EP & 4LD \\
\hline $\begin{array}{l}\text { No knowledge } \\
\begin{array}{l}(665 \text { instances }) \\
\text { Knowledge }\end{array}\end{array}$ & 77 & 84 & 48 & 40 & 44 & 23 & 27 & 41 \\
$\begin{array}{l}(266 \text { instances }) \\
\text { Difference }\end{array}$ & 10 & 4 & -3 & 6 & 6 & 2 & 1 & 2 \\
p-value & 0.00 & 0.02 & 0.13 & 0.00 & 0.00 & 0.12 & 0.20 & 0.20 \\
\hline \hline
\end{tabular}

Table 14. Average points obtained with and without knowledge of opponents' preferences. We report the pvalue for a t-test testing the null hypothesis that the difference is zero.

\begin{tabular}{lllllllll}
\hline \hline & 2ACC & 2SCC & 3DS & 3LD & 3SC & 4DS & 4LD & 4EP \\
Round 1 & 68 & 81 & 50 & 34 & 40 & 23 & 34 & 25 \\
Round 5 & 82 & 91 & 50 & 45 & 44 & 24 & 45 & 28 \\
\hline Difference & 14 & 10 & 0 & 11 & 5 & 1 & 8 & 3 \\
p-value & 0.00 & 0.00 & 0.97 & 0.00 & 0.05 & 0.66 & 0.01 & 0.07 \\
\hline
\end{tabular}

Table 15. Average points obtained in rounds 1 and 5 . We report the p-value for a t-test testing the null hypothesis that the difference is zero.

\begin{tabular}{lllllllll}
\hline \hline & 2ACC & 2SCC & 3DS & 3LD & 3SC & 4DS & 4LD & 4EP \\
\hline Round 5 & 82 & 91 & 50 & 45 & 44 & 24 & 45 & 28 \\
Round 7 & 89 & 91 & 46 & 47 & 50 & 26 & 46 & 29 \\
\hline Difference & 7 & 0 & -4 & 2 & 6 & 2 & 0 & 1 \\
p-value & 0.04 & 0.89 & 0.20 & 0.51 & 0.02 & 0.30 & 0.96 & 0.58 \\
\hline \hline
\end{tabular}

Table 16. Average points obtained in rounds 5 and 7 . We report the p-value for a t-test testing the null hypothesis that the difference is zero. 